\title{
Microfluidics-based systems biology
}

\author{
David N. Breslauer, Philip J. Lee and Luke P. Lee \\ Received 3rd November 2005, Accepted 23rd December 2005 \\ First published as an Advance Article on the web 9th January 2006 \\ DOI: $10.1039 / \mathrm{b5} 15632 \mathrm{~g}$
}

Systems biology seeks to develop a complete understanding of cellular mechanisms by studying the functions of intra- and inter-cellular molecular interactions that trigger and coordinate cellular events. However, the complexity of biological systems causes accurate and precise systems biology experimentation to be a difficult task. Most biological experimentation focuses on highly detailed investigation of a single signaling mechanism, which lacks the throughput necessary to reconstruct the entirety of the biological system, while high-throughput testing often lacks the fidelity and detail necessary to fully comprehend the mechanisms of signal propagation. Systems biology experimentation, however, can benefit greatly from the progress in the development of microfluidic devices. Microfluidics provides the opportunity to study cells effectively on both a single- and multi-cellular level with high-resolution and localized application of experimental conditions with biomimetic physiological conditions. Additionally, the ability to massively array devices on a chip opens the door for high-throughput, high fidelity experimentation to aid in accurate and precise unraveling of the intertwined signaling systems that compose the inner workings of the cell.

\section{Introduction}

The post-genomic era has ushered in the promise of complete understanding of biological systems. Systems biology is the discipline currently leading this endeavor, attempting to elucidate the myriad of interactions between the dynamics of molecular components in a biological system such that system behavior can be accurately predicted. ${ }^{1}$ To achieve this goal, dynamic models of biological systems must be developed. The approach to model development, as described by Ideker et al., ${ }^{2}$ is the use of existing data to formulate an initial model and the subsequent use of the model to predict system responses to various perturbations. These perturbations are then experimentally applied to the system and the collected results are used for further model refinement. An example systems model

UCSF/UC Berkeley Bioengineering Graduate Group, Department of Bioengineering, University of California Berkeley, Berkeley, CA, USA. E-mail:lplee@berkeley.edu of epidermal growth factor receptor (EGFR) signaling is shown in Fig. 1.

Due to the overwhelming size and complexity of biological systems, high-throughput experimental methods are generally employed to generate the immense amount of information required to sufficiently describe a system. For example, methodologies such as Yeast Two-Hybrid (Y2H) screens ${ }^{4}$ and technologies such as protein microarrays ${ }^{5}$ return largescale data sets of protein interactions. The limitations faced by such high-throughput methods are due to the lack of whole cell experimentation, leading to potential false positives from molecules that may not truly interact in vivo because of a lack of spatial proximity, organelle compartmentalization, or regulation by other components. These high-throughput techniques generally provide no data regarding the interaction dynamics between molecules, either. Methods such as affinity chromatography and protein fluorescence are used to quantify interaction dynamics (e.g. determine binding constants and

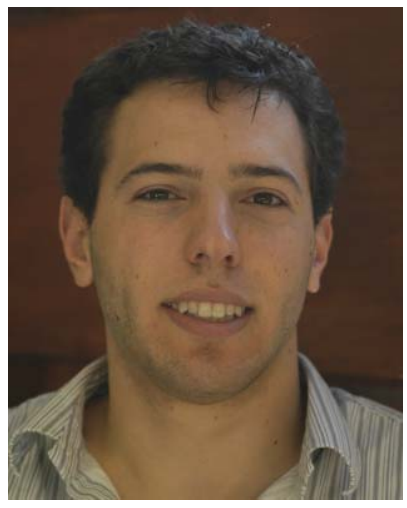

David N. Breslauer
David Breslauer is a graduate student in the UCSF/UC Berkeley Bioengineering graduate group. His research interest is primarily in the use of novel micro-scale technologies for the quantitative study of cellular signaling and communication. David earned his BS in Bioengineering from the University of California, San Diego in 2005.

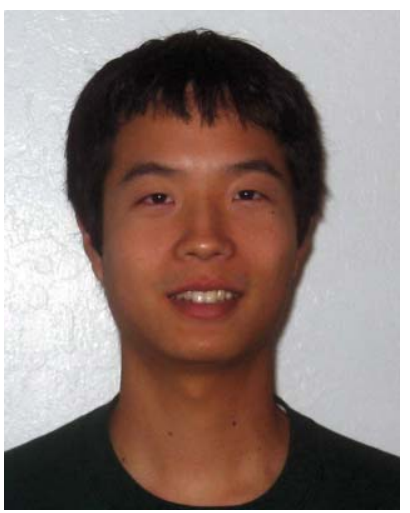

Philip Lee is currently a graduate student in the UCSF/UC Berkeley Bioengineering program. His primary research focus is the application of microtechnologies to improve in vitro cell experimentation. Philip received undergraduate degrees in Chemical Engineering and Biology at the Massachusetts Institute of Technology in 2003. 


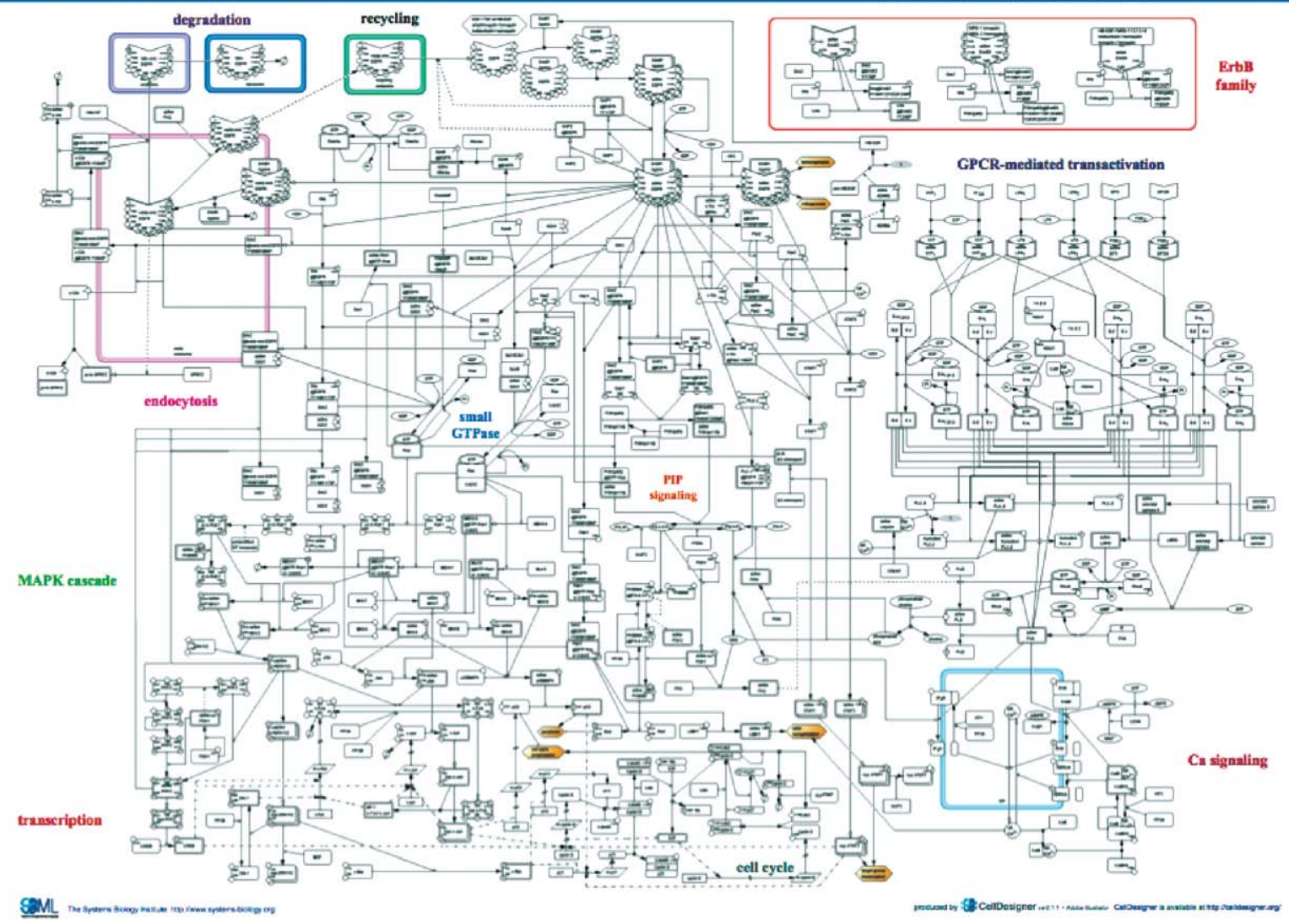

Fig. 1 A recently developed comprehensive map of EGFR signaling. ${ }^{3}$ The entire map of the EGFR pathway is quite large but demonstrates how intracellular components interact to trigger events.

diffusion coefficients), ${ }^{6}$ but are generally not applied in a highthroughput manner. DNA microarrays generate an enormous

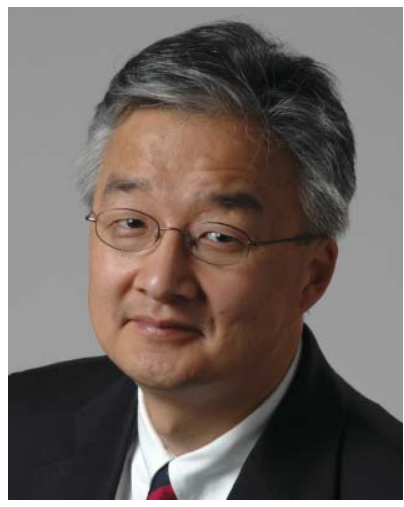

Professor Luke P. Lee is Lloyd Distinguished Professor of Bioengineering at UC Berkeley. He is also Director of the Biomolecular Nanotechnology Center and Co-Director of the Berkeley Sensor and Actuator Center. He received both his $B A$ in Biophysics and PhD in Applied Physics with a minor in Bioengineering from UC Berkeley. His current research interests are quantitative biomedical

Luke P. Lee wealth of information regarding gene expression levels, but only provide a static snapshot of the sampled state of cells. Additionally, many techniques used to determine the dynamic parameters of biochemical interactions suffer from poor temporal resolution, calculating parameters from $10^{2} \mathrm{~s}-10^{4} \mathrm{~s}$ scale measurements rather than $10^{-3} \mathrm{~s}-10^{0} \mathrm{~s}$ scale at which these interactions occur. ${ }^{7}$ Consequently, there is a need for new instrumentation that can perform high-throughput whole cell experimentation under a large variety of highly specific environmental conditions and at a cellular scale.

Microfluidic technologies have the potential to significantly progress systems biology by overcoming the limitations hindering the currently available instrumentation. Taking advantage of the same microfabrication techniques used to pattern integrated circuits on silicon wafers, microfluidics is the design and development of miniaturized fluidic devices that manipulate samples at nanoliter volumes. Due to its extremely small-scale and low cost of production, microfluidics can revolutionize our ability to perform procedures such as highthroughput drug screening, biological and chemical sensing, and genetic analysis. ${ }^{8}$ For example, the ability to process samples at nanoliter volumes significantly reduces dosage requirements for testing, cutting down on both cost and waste. The size of microfluidic systems increases the speed of common experimental techniques, such as protein and DNA electrophoresis, by orders of magnitude. ${ }^{9}$ Additionally, the 


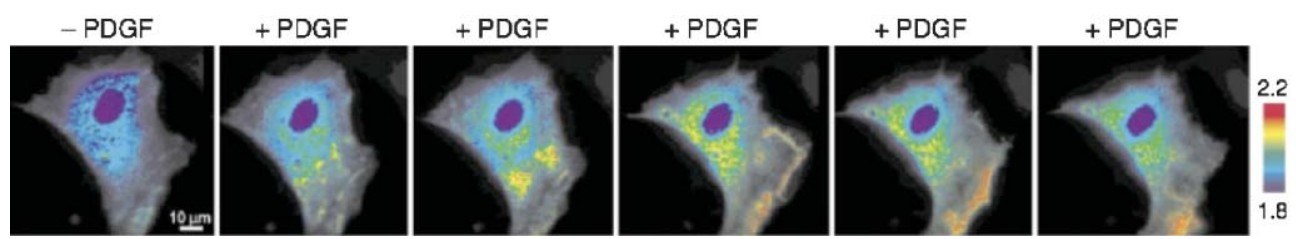

Fig. 2 Fluorescence imaging of the distribution of Abl tyrosine-kinase activity in a NIH 3T3 cell before and after perturbation with plateletderived growth factor (PDGF). ${ }^{12}$

small form factor of microfluidic components allows for the integration of functionally diverse systems on a single miniaturized device. Referred to as micro-Total Analysis Systems ( $\mu$-TAS) or Lab-on-a-Chip (LOC), these devices perform techniques such as cell culturing, Fluorescence Activated Cell Sorting (FACS) ${ }^{10}$ and Polymerase Chain Reaction $(\mathrm{PCR})^{11}$ on one small and disposable chip.

In combination with the recent advances in molecular fluorescent imaging technology, ${ }^{12}$ microfluidics presents unique opportunities for live cell experimentation. An example of fluorescent imaging techniques for studying signaling networks is shown in Fig. 2. These techniques can be performed within microfluidic devices, benefiting from the small-scale of microfluidics, which allows for highly specific control or perturbations of the cellular microenvironment, as well as subcellular manipulation of molecules. ${ }^{13}$ Additionally, most microfluidic devices can easily be scaled to perform massive-scale experimentation without impacting fabrication cost. Finally, using high-throughput microfluidics, visualization of molecular movement within whole cells allows for the large-scale determination of dynamic properties, including temporal and spatial characterizations, a dimension only recently gaining popular consideration in the development of mathematical models of biological systems. ${ }^{14-16}$

Currently, the biggest roadblock in the large-scale application of microfluidic engineering to systems biology is the lack of experimental compatibility with established methodologies. While the current focus of the field is in validating the fundamental advantages of microfabricated systems, the next stage of development needs to address the engineering of functional instrumentation to exploit the novel advantages noted above. Primarily, the micro/macro interface is an area that deserves increased attention. For example, most microfluidic devices lack an acceptable methodology for low volume delivery of cells/reagents, making the claims of high throughput and reagent savings unattainable in practice. Similarly, customized detection methodologies for the low concentrations in microfluidic channels have yet to be reliably introduced. As demonstrated in this review, the initial progress in microengineering for biological studies is reaching a critical mass, with substantial technological improvement expected in the next 5-10 years.

This review highlights relevant developments in microfluidic devices and microfluidic biological experimentation from the perspective of their use toward the advancement of systems biology. It begins with a summary of the basic knowledge required to understand microfluidics and an overview of several important points to consider when planning systems biology experiments. It is then organized into categorical sections that contain discussions on microfluidic devices that have been developed and the experiments that have been performed using them. This review concludes with an outline of how microfluidic technologies might be utilized to develop a cellular systems biology platform.

\section{Micro-scale biophysics}

Upon shrinking to the micro-scale, the dominant forces of physical phenomena change. Three characteristics of physics at the micro-scale (laminar flow, diffusion, surface area to volume ratio) are briefly explained, as they are the most pertinent to the microfluidic devices reviewed. More exhaustive discussions of micro-scale physics are available from other sources. ${ }^{8}$

Due to the miniscule characteristic lengths of microfluidic channels (down to the order of $1 \mu \mathrm{m}$ ), microfluidic flow is generally laminar. Turbulent flow is chaotic, whereas laminar flow allows precise calculation of mass transport as a function of time. The convective flow profile for laminar flow is straightforward to calculate from parameters such as channel geometry, pressure drop, and fluid properties. Due to the translation invariance of laminar flow, fluid streamlines remain constant over time, and mixing of microfluidic streams occurs primarily by diffusion.

By their natural Brownian movement, particles tend to spread such that they are evenly distributed over a volume. The time it takes a particle to travel, one-dimensionally, over a distance is directly proportional to the square of that distance. Consequently, for large distances, diffusive motion occurs on a high enough timescale to generally be negligible. When operating on the micro-scale, however, diffusion can account for a significant portion of mass transport.

When moving to the micro-scale, the relative amount of surface area exposure experienced by a volume of fluid greatly increases. Increased surface area contact causes faster diffusion and increased heat conduction. Macromolecules are easily adsorbed to device materials such that the effects of diffusion and adsorption onto the device surface must be accounted for.

These first two properties (laminar flow, controlled diffusion) enable the creation of unique experimental platforms using microfluidics. Laminar flow allows barrier-free adjacent perfusion of different reagents, which can be used for localized treatment of cells. Controlled diffusion and laminar flow in combination can be used to construct chemical gradients with sub-cellular resolution. The high surface-to-volume ratio of microfluidic channels, however, presents a serious design consideration due to the potential absorption of certain molecules into the device material. 


\section{Considerations for studying biological systems}

Systems biology technology is a relatively young field of study and has few standardized methodologies for experimentation. The following are several points to bear in mind when planning experimentation on biological systems.

Because tissues are composed of a heterogeneous cellular matrix, cell population studies are perhaps more physiologically relevant than single-cell experimentation. The cells within a population, however, are not homogeneous. When performing experimentation on an ensemble of cells, the measurement of the individual cellular response is lost in the bulk average response. Experiments have shown that significant cellular phenotypes are only resolvable when studying a single cell, ${ }^{17,18}$ indicating the importance of studying individual cells rather than only heterogeneous populations.

Intracellular signaling systems are complex networks of interactions that respond to environmental cues. These environmental cues, however, could likely be signals from other cells. Whereas understanding how a cell functions on its own is important, the interplay between cells can significantly alter behavior. In order to account for intercellular signaling, cells can be studied in populations, leading to the dilemmas discussed above, or in systems with shared media.

Lastly, whereas protein networks are often considered a large nodal network with massive amounts of connections, ${ }^{19}$ additional factors that should be considered may regulate the functionality of molecular systems. Temporally, not all interacting factors are expressed at the same time, despite the physical ability to interact. Spatially, organelle compartmentalization can place a barrier between potential interacting partners, localized receptors limit a cells ability to sense a trigger, and diffusion imposes restrictions on the speed at which interacting molecules can reach each other.

Studies of cellular signaling systems are often performed on cell cultures, using the lysate for procedures such as protein electrophoresis or DNA microarrays. The microfluidic platforms reviewed herein offer the option of both cell culture and single cell experimentation, enabling the elucidation of precise molecular mechanisms from isolated cells or those in more physiologically relevant multi-cellular environments. Additionally, with the use of optical probes, signaling components can be spatiotemporally characterized to determine the in vivo dynamics of molecular interactions.

\section{Microfluidic components and devices for biological experimentation}

\subsection{Exploiting microfluidic flow for unique opportunities in experimentation}

In order to model a biological system, it is important to understand its dynamic output in response to a specific input. Systematic network perturbation has been a popular and successful means of generating data of system responses. The data is then statistically correlated for reconstruction of the pertinent biological networks. ${ }^{20-23}$ When applying systematic perturbations, high-specificity stimuli are required in order to isolate the resulting system response. Contemporary laboratory techniques saturate cell cultures in reagents in order to subsequently study their responses. Temporal variation of stimuli is limited by the timescale at which the experimenter can replace reagent and localized stimuli is only achieved through the manual manipulation of glass micropipettes. Microfluidics technology enables high-specificity perturbation of many parallel cellular microenvironment spaces. Laminar flow can be used to apply precise, localized, as well as spatially and temporally dynamic perturbations.

As a demonstration, Takayama et al. ${ }^{13,24}$ adhered a bovine capillary endothelial (BCE) cell in a microfluidic channel and flowed Trypsin/EDTA locally over it, observing the detachment of the treated area. The simple microfluidic device consisted of three inlets from which the flows converged into a rectangular capillary as parallel laminar streams. Different color fluorescent dyes were flowed over opposite sides of a live BCE cell, staining two subpopulations of mitochondria for observation (Fig. 3c,d). Subsequently, the cell was locally treated with latrunculin A (promoting the break up of
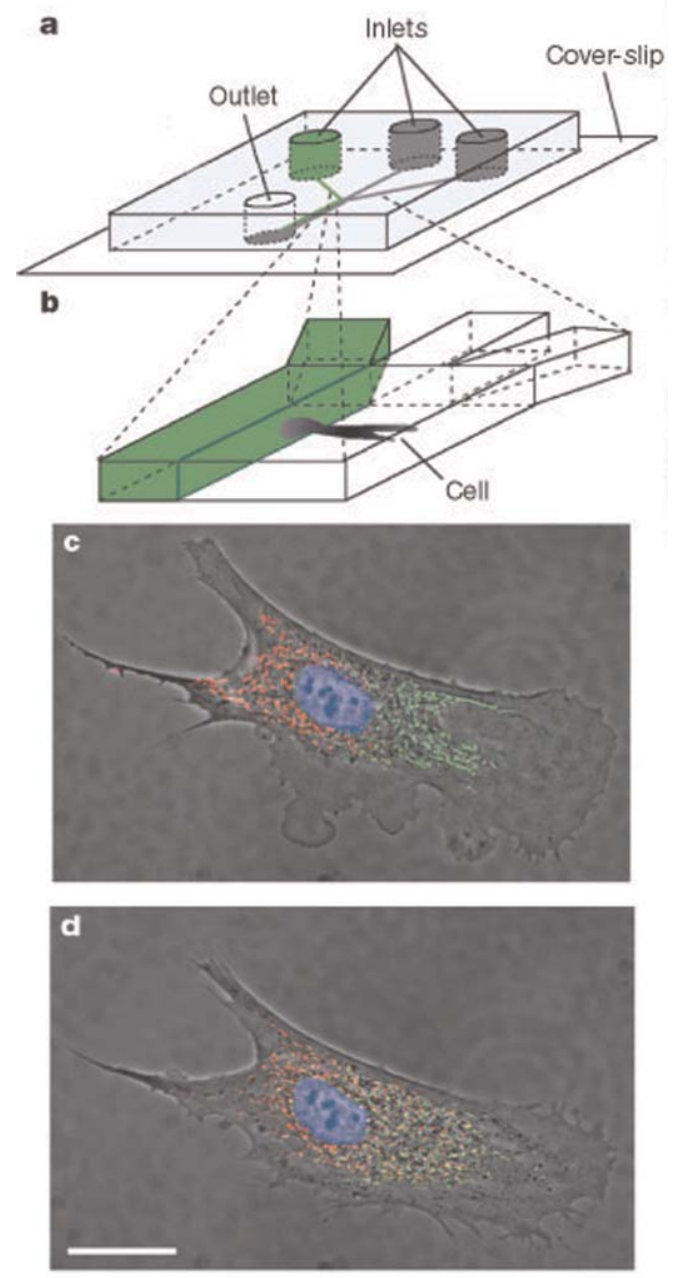

Fig. 3 Experimental design of PARTCELL. The inlets and outlets where the three input streams converge is magnified $(a, b) .{ }^{13}$ BCE cell after treatment of the right side with Mitotracker Green FM and the left side with Mitotracker Red $\mathrm{CM}-\mathrm{H}_{2} \mathrm{XRos}$ in order to observe the movement of subpopulations of mitochondria. The image on the right was taken $2.5 \mathrm{~h}$ later to demonstrate mitochondrial movement (c, d). ${ }^{13}$ 
polymeric actin filaments) and the mitochondrial response to the cellular damage was observed. Dubbed "PARTCELL" by the investigators, the method allows for local cellular delivery of membrane-permeable molecules, maintaining localized flow both inside and around the cell (Fig. 3a,b).

The necessity for spatially localized stimuli in the study of molecular signaling systems was demonstrated through the investigation of intracellular spatiotemporal propagation of localized epidermal growth factor (EGF) stimulation using microfluidics. ${ }^{25}$ The EGF-receptor (EGFR) system is well characterized with much success derived from the use of systems-level computational modeling in its investigation. ${ }^{26} \mathrm{~A}$ previous study had reported that local EGF stimulation caused propagation of receptor phosphorylation over the entire cell. However, to enable visualization of the cellular events, EGFR had to be overexpressed. Using microfluidics, Sawano et $a .^{25}$ were able to study EGF stimulation without altering EGFR density and found contradictory results. They discovered that EGF signaling remains local when EGFR is expressed at a normal level (Fig. 4); however, when the receptors are overexpressed as in the previous study, EGF signaling propagates over the entire cell. The experiment took into account the spatial context of cellular signaling and only recently has spatial heterogeneity been seriously gaining momentum as a necessary dimension to consider in systems biology modeling and simulation. ${ }^{12,14-16}$

On a cellular systems scale, Lucchetta et l. $^{27}$ used localized microfluidic flow to explore embryonic patterning compensation mechanisms against spatiotemporal perturbations applied to a Drosophila embryo. The robustness of the Drosophila patterning system to a large range of physicochemical parameters has been well-demonstrated using system-level mathematical models. ${ }^{28,29}$ Luccetta et al. tested these mechanisms with an embryo positioned between two laminar streams of different temperatures. Dynamic Even-skipped gene expression, which fluoresces a well-known striped pattern, was one factor studied (Fig. 5). Stripe positions remained consistent with control experiments, although stripes appeared in a different order than normal. Dynamic Hunchback gene intensity profiles were recorded in response to experiments during which the temperature step was temporarily reversed. Hunchback expression variability increased most significantly during a temperature reversal between $65 \mathrm{~min}$ and $100 \mathrm{~min}$, indicating that this development period is the most critical for compensatory mechanism activity. Mathematically, von Dassow et al. ${ }^{28}$ demonstrated that the Drosophila segment polarity network is robust due to the intra- and inter-cellular topology of the system. To this end, an entire Drosophila protein-protein interaction map has been developed. ${ }^{30}$ Additionally, many other specific Drosophila systems are available (e.g. circadian rhythm, ${ }^{31} \mathrm{EGFR}^{32}$ ) whose study could benefit from the full experimental capabilities of microfluidics.

Because cells are responsive to concentration differences down to $2 \%$ across their diameter, ${ }^{33}$ the ability to generate gradients with single-cell resolution is extremely beneficial for the study of chemotaxis and polarized signaling. Spatial complexity and localization in intracellular systems is important in chemotaxis signaling ${ }^{34}$ and could thus benefit greatly from the high-specificity of microfluidics. Furthermore, cellular signaling during chemotaxis has already been extensively characterized ${ }^{34-36}$ and very successfully modeled. ${ }^{37-39}$ Modeling approaches to chemotaxis signaling have demonstrated robustness $^{38}$ and adaptation ${ }^{37}$ as two properties of the bacterial chemotaxis signaling system. Studies of adaptation would be well suited for microfluidic gradient generators because of their ability to maintain basal concentrations across the gradient.

Jeon et al. $^{33}$ developed a microfluidic device capable of creating a single cell-resolution concentration gradient of three input streams. Initially, a network of serpentine channels allowed adjacent laminar streams to flow together long enough
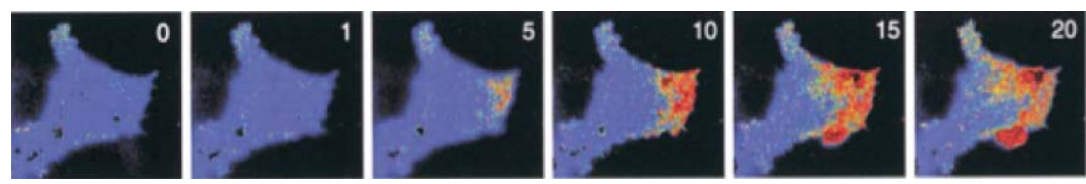

Fig. 4 Time-series (min) response of microfluidic local Rhodamine-EGF stimulation through visualization of Ras activation. The intensity of the response ranges from low (blue) to high (red). ${ }^{25}$
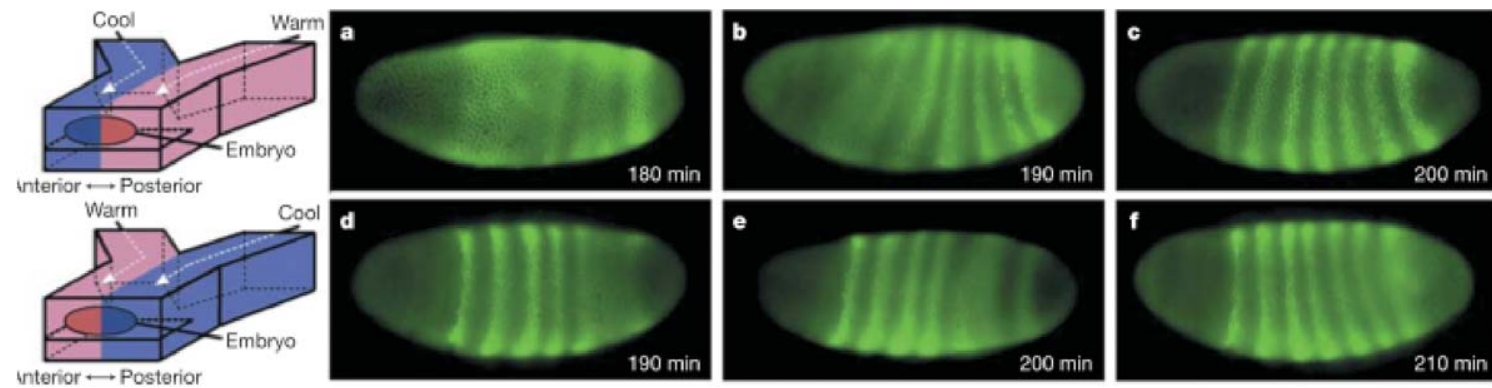

Fig. 5 Experimental setup of the microfluidic device used to apply a temperature across a Drosophila embryo and the dynamic Even-skipped gene expression response. The second row shows the reversed T-step experiment of the first experiment. Even-skipped expression was faster in the warm half of the embryo, but both embryos resolved the same final expression pattern. ${ }^{27}$ 
for full diffusive mixing. By organizing these serpentine channels in a pyramidal arrangement, as in Fig. 6, fluid flows could be joined, mixed, and then divided. Streams of different concentrations were flowed adjacently into a single outlet, creating a concentration gradient in the outlet. If the bottomlevel output channels are not converged into a single outlet, the device could be used in order to create and isolate several differently concentrated mixtures of the input streams. ${ }^{40,41}$ The gradient shape was adjustable in width and shifted from side to side by changing the individual input stream flow rates, which can be performed dynamically. With little modification, linear and nonlinear concentration gradients that could be controlled temporally and spatially were generated. ${ }^{42}$ The slope, baseline, and direction of linear gradients as well as the power of a power law form gradient were variable. Notably, the ability to control the baseline of the gradient is important if an experiment requires a minimum background concentration. Additionally, sawtooth profiles, periodic profiles, and overlapping gradients of different solutes can be produced. ${ }^{43}$

Neutrophil chemotaxis in interleukin-8 (IL-8) gradients was investigated using the gradient device. ${ }^{44}$ The investigators observed neutrophils in a linear, hill, and cliff (increase and sudden drop to zero) gradient of IL-8. In the linear gradient, neutrophils migrated toward increasing concentrations of IL-8 (Fig. 7). In the cliff gradient, neutrophils ceased migration upon reaching the sudden drop to zero. However, in the hill gradient, neutrophils overshot the maximum concentration of IL-8. After the overshoot, they reversed direction back toward maximum concentration. Another microfluidic experiment determined that the mean concentration of IL-8 across the gradient (optimally $3 \mathrm{nM}$ ) was the most influential factor in directing effective neutrophil chemotaxis. ${ }^{45}$ Such high-precision data provides unique opportunities to thoroughly validate models of the chemotactic signaling pathway. Additionally, the study of optimum mean concentration generates interesting data for use in studies of system adaptation.

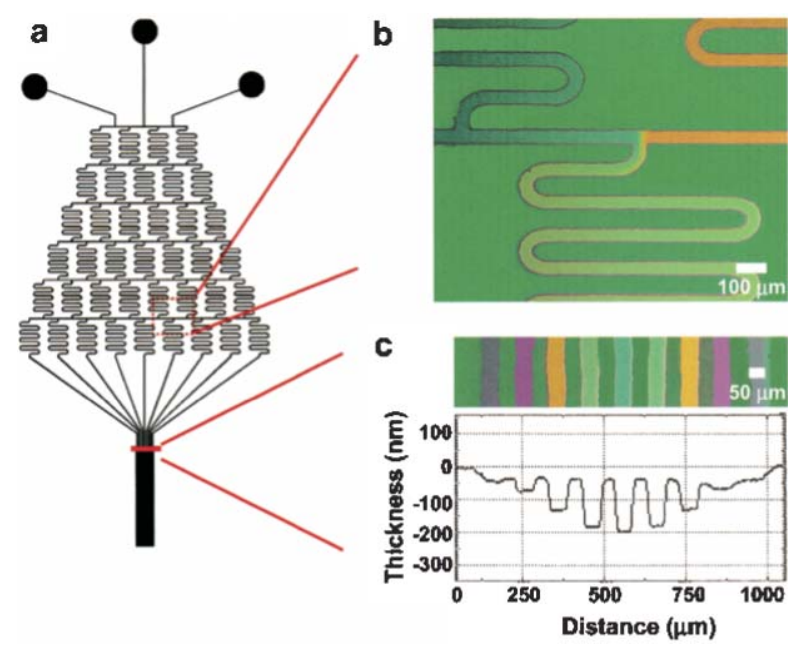

Fig. 6 (a) Design of the pyramidal gradient generator. (b) Each serpentine channel splits and then joins with another channel. (c) Each flow is a different concentration of the inputs, isolated in separate channels before being combined into a single gradient stream. ${ }^{33}$
A
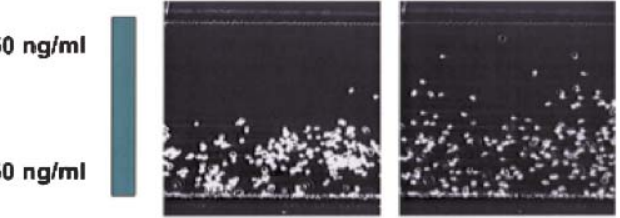

B
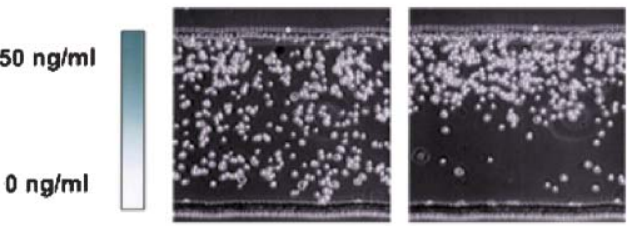

C
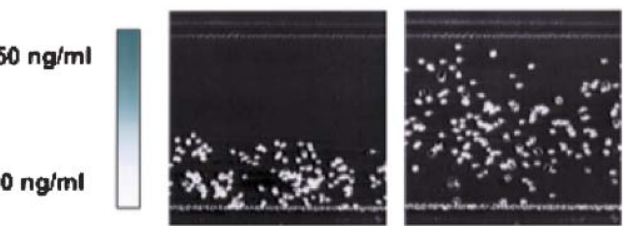

D

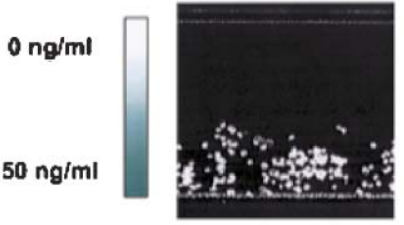

$0 \min$

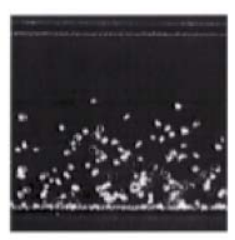

$90 \mathrm{~min}$
Fig. 7 Neutrophil chemotaxis over linear gradients of IL-8. Neutrophils tended toward increasing IL-8 concentration. ${ }^{44}$

Breast cancer cell chemotaxis in EGF gradients has also been studied. ${ }^{46}$ Although previous reports had indicated that breast cancer cells migrated toward EGF, no evidence of directed chemotaxis in a linear gradient was found. However, when polynomial gradients that mimicked the experiment in the previous reports were applied, significantly directed chemotaxis toward increasing EGF concentration was observed. Just as in the experiment on EGFR localization, the use of microfluidics in this case eliminated confounding experimental conditions. Theoretical models of cells in the different gradient profiles indicated that the difference in the fraction of receptors occupied between the front and back of the cell in halfway through a gradient was significantly greater in the nonlinear gradient than in the linear gradient. Once again, precision microfluidic experiments eliminated confounding variables that significantly altered cellular response.

Even though continuous gradients can be created with high levels of accuracy, the ability to expose cells to many dilutions of molecular solutions in parallel also enables high-throughput screening of cellular response to different environments. Neils et $a l^{47}$ created a microfluidic combinatorial mixer that had four inputs and outputted 16 different mixtures. More specifically than the ability to perform multiple simultaneous experiments, studying cells under a variety of dilutions facilitates the elucidation of input thresholds for triggering system responses as well as the study of stimulus amplification throughout a signaling cascade. 
Laminar flow and controlled diffusion obviously present new opportunities for biological experimentation. In their most simplistic use, they allow dynamic and localized cellular perturbation. Furthermore, they enable the creation of multiplexed chemical dilutions and high-resolution gradients. As shown, these benefits have been directly realized for signal transduction and chemotaxis studies. However, as will be shown, integrating these properties with constant perfusion has allowed the creation of far more extensive devices that can perform a wide variety of molecular- and cellular-level experimentation.

\subsection{Experimental platforms for cellular analysis and cellular systems biology}

The devices discussed above cover the basis for perturbing systems using microfluidics. However, the ability to further manipulate individual cells expands the possibilities for experimentation. Whereas the biomolecular detection platforms used in systems biology experimentation have significantly advanced as the field has grown, methods of cellular perturbation and analysis have not particularly progressed past standard laboratory techniques. The following devices, combined with optical probes or integrated microfluidic biomolecular analysis devices, provide novel platforms with which to perform molecular and cellular systems biology experimentation.

Wheeler et al $^{48}$ developed an elegant device for the study of single cells. The device trapped a single cell, separating it from an ensemble, in a microfluidic T-junction. The design minimized the pressure drop over the cell as it was trapped, decreasing the mechanical stress to which the cell was exposed. After a cell was trapped, reagent was delivered by flowing it toward the cell. However, by delivering a flow from the side of the T-junction, the extra flow acted as a wall between the reagent and the cell. Calcium flux response to cross-linking by performing two-step perfusion was studied. Cross-linking is the result of certain intracellular signaling cascades being stimulated in response to the binding of multiple ligands to linked receptors (in this case, $\mathrm{Fc}_{\gamma} \mathrm{R}$ ). Initially they perfused a cell with human immunoglobulin $\mathrm{G}$ ( $\mathrm{IgG}$ ) and subsequently with goat anti-human IgG. Dynamic fluorescence of the cell during the second perfusion was recorded, noting the lag time between secondary perfusion and the system response as well as the duration of the response. Both of these types of measurements are useful in fitting computational models to experimental data, with the platform itself providing a novel means of studying single cell gating kinetics.

Whereas single cell investigation provides critical insight into intracellular systems, as discussed, the study of multicellular systems is of significant physiological relevance. Cellcell communication has influence on the cellular life cycle, and multicellular systems maintain intercellular signaling upon experimentation. However, in order to investigate the effects of intercellular communication on signaling systems, it is most beneficial to isolate a cell pair, the smallest unit of study for cell-cell communication. Lee, Hung et al. ${ }^{49}$ developed a unique device to study gap junction-facilitated intercellular signaling between arrays of cell pairs, with single-cell resolution. Two cells were held in opposing trapping channels such that their membranes were in contact. Commonly, fluorescently dyed cells are cultured with unlabeled cells and the transfer of dye is used to determine whether cells have developed intercellular gap junctions. This device is an improvement over such techniques, as it enables the study of single-cell transfer kinetics with high precision because the time and location of the cell-cell contact is known, eliminating any arbitrariness in accounting for the basis of cellular communication.

Isolated single cell and cell pair experimentation have been demonstrated; however, Chin et al. ${ }^{50}$ developed a massively high-throughput single cell array that maintained paracrine signaling throughout experimentation. The microfabricated array contained approximately 10000 microwells and the investigators were able to track over 3000 individual rat neural stem cells. All of the wells were exposed to the same common media and consequently cells were able to draw paracrine signaling factors released from neighboring cells. This enabled single cells to survive and proliferate more easily, indicating the impact of intercellular signaling systems in the mechanisms that control the cellular life cycle.

Davidsson et al. ${ }^{51}$ investigated the effect of paracrine diffusion distances and stress in their microfluidic cellular analysis device. The investigators created a microfluidic system that allowed long-term cellular analysis in sterile conditions while also minimizing the stress imposed on the cell. Because cellular signaling systems respond to stress, any decrease in extraneous stress allows more accurate measurements of specific signals than otherwise. The device consisted of parallel $\mathrm{V}$-shaped grooves that increased the area for cell attachment while decreasing the distance between cells and consequently decreasing diffusion times between them. Long-term monitoring of cells was achieved for up to 30 hours. Part of the utility of long-term monitoring was that the investigators were able to study the response of cells upon placement into the device and allow the cells to normalize to their new environment before experimentation. Using the HFF11 cell line, the investigators dynamically quantified Photinus luciferase (LUC) expression after placement of cells into the chamber and after allowing them to adjust to their new environment. They found a significant increase in LUC expression directly after loading the cells into their device. After 30 mins of stimulation LUC expression began in comparison to six hours in a microtiter plate assay. They attributed this decrease in response time to the decreased diffusion distances and the continuous flow of fresh cell medium in the microfluidic device. The short stimulation time makes the device useful for real-time study of intracellular signaling kinetics. The investigator's characterization of the device is unique because, as mentioned, any reductions in the imposed stress on cells will reduce the activity of stress-response pathways.

To the same end, Lee et $a l .{ }^{41}$ developed a high-throughput microfluidic cell culture device that greatly decreased shear stress experienced by cells in culture by effectively mimicking physiological interstitial space. It was an 8 by 8 array of culture wells with individually addressable rows and a gradient generator across the columns (Fig. 8a,c). The success of the design was due to its ability to isolate the cultures from convective flow and supply them with nutrients almost entirely 

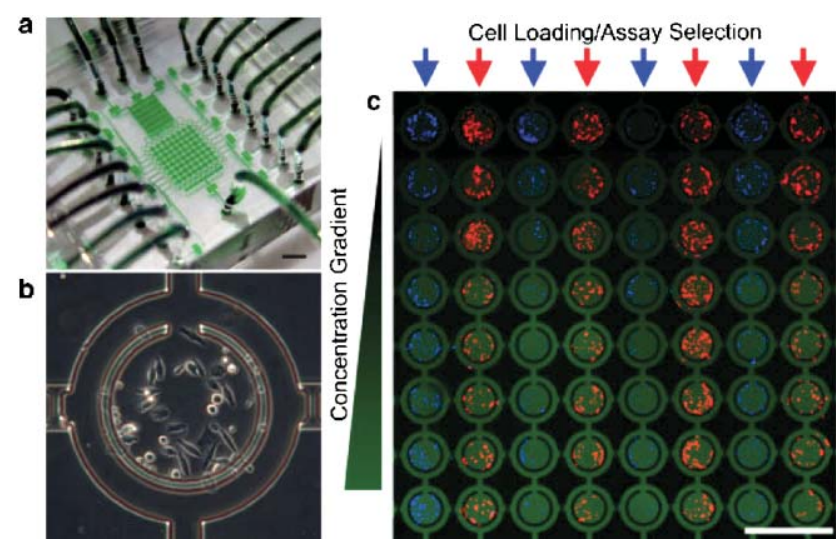

Fig. 8 (a) Entire cell culture device stained with green dye for visualization. (b) Close up of HeLa cells growing in a single culture chamber. (c) 8 by 8 cell culture grid. A concentration gradient of media was perfused across the device (left to right). Individual column addressability is demonstrated by nuclei staining (blue) and cytoplasm staining (red). ${ }^{41}$

through diffusion. The device was able to culture a variety of cell lines, including primary cells (Fig. 9). They added a gradient generator in order to create a concentration gradient across the culture chambers, enabling experimentation under a variety of conditions without any increased sample preparation time. Although an 8 by 8 array of cell culture chambers was demonstrated, the device could easily be up-scaled for increasingly high-throughput experimentation. With the addition of microfluidic valves, the device could be modified such that each culture chamber is individually addressable, essentially creating 64 entirely unique cell culture environments.

Balagaddé et al. ${ }^{52}$ cleverly demonstrated the utility of a microfluidic cell culture device for cellular systems biology.
They developed a microfluidic bioreactor, composed of six independent $16 \mathrm{nl}$ reaction chambers that eliminated bacterial wall growth, avoiding interference within the culture from bacterial biofilms. The investigators measured the population dynamics of bacteria modified with an inducible synthetic system for population control in the device. Synthetic biology is the design and engineering of custom biological systems for specific tasks, and is a field intimately tied up with systems biology. ${ }^{53}$ The synthetic circuit introduced into these bacteria dynamically regulated cell density by controlling cell death (through expression of $l a c Z \alpha-c c d B$ ) in response to detection of the intercellular signaling molecule acylhomoserine lactone. They observed five modified populations of bacteria (three with the circuit on, two with it off) and one control population with no circuit. The populations with the induced circuit grew in an oscillatory fashion in comparison to the exponential growth of the other populations. The oscillatory results fit the theoretical mathematical model of synthetic population dynamics. An interesting consequence of the small size of the reaction chambers is that the synthetic circuit was stable over longer periods than in a macro-scale culture. Population control was maintained for over $500 \mathrm{~h}$, with stability attributed to the fact that the micro-scale chambers maintained a population size from approximately $10^{2}$ to $10^{4}$ cells in comparison to macro-scale cultures with a population size of $10^{9}$ cells. Theoretically, the lower population size reduced the rate of mutations in the overall population that could otherwise have eventually dominated the bacterial culture. Consequently, small reaction chambers and population sizes could provide beneficial uniformity for cellular studies.

Despite the elegance of such cellular level studies, systems biology is based on the understanding of the exact molecular mechanisms behind cellular processes. The Living Cell Array (LCA) is an exquisite representation of the capabilities of
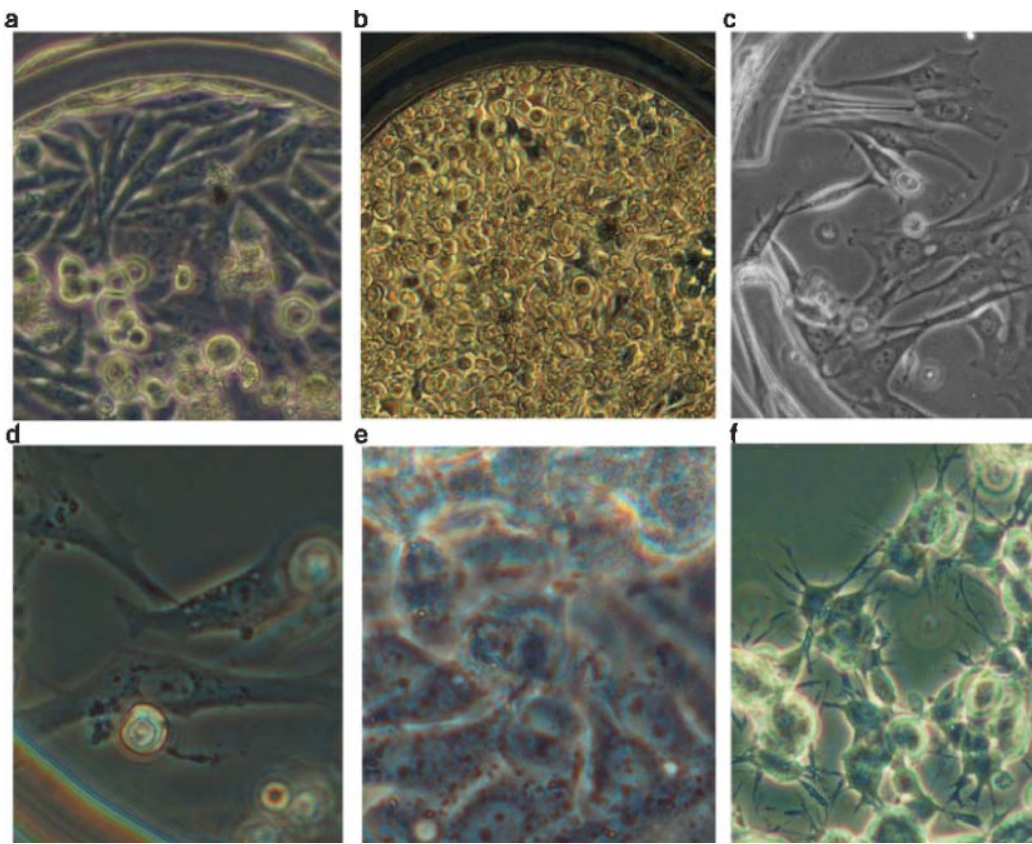

Fig. 9 A variety of cell types growing in the cell culture device. (a) HeLa. (b) High density HeLa after extended perfusion. (c) NIH3T3 fibroblasts. (d) Primary bovine endothelial cells. (e) HepG2 hepatocytes. (f) Human SY5Y neuroblastoma. ${ }^{41}$ 
microfluidic devices for systems biology; it is a device capable of high-throughput, dynamic, whole cell gene expression profiling. ${ }^{40}$ LCA consists of the pyramidal gradient generator $^{33}$ that flows different concentrations of stimulus in medium through each column of an array of cell culture chambers. By seeding the device with cells containing a green fluorescence protein (GFP) reporter attached to a target gene, fluorescence levels representative of gene expression were dynamically recorded (Fig. 10). The investigators perfused cells with different concentrations of the cytokine TNK- $\alpha$, while observing the expression dynamics of the transcription factor NF$\kappa \mathrm{B}$. The average fluorescent intensity per cell was recorded over time for the different cytokine concentrations. The doseresponse kinetics was similar for each concentration, and increased dosage prompted greater magnitude responses. Whereas they found that results of the extreme high and low dosages were highly reproducible, significant variability was recorded in response to intermediate doses due to the heterogeneity of the cell population, "highlighting the importance of single cell measurements."

The platforms discussed are distinctive in their ability to perform experiments on cells from a single cell to a full culture level. Single cell platforms allow for the study of individual signaling systems that are otherwise indistinguishable within multicellular systems. Microfluidic devices can easily trap single cells and perform large-scale studies of them within isolation, or under the influence of singular gap-junction facilitated or paracrine signaling. Microfluidic cell culture devices provide low-stress environments to minimize shockinduced distortion of intracellular systems as well as nanoliter size chambers that limit population size and thus mutation rate. However, extracting, manipulating, and assaying nanoliter volumes is difficult to perform manually and is likely to

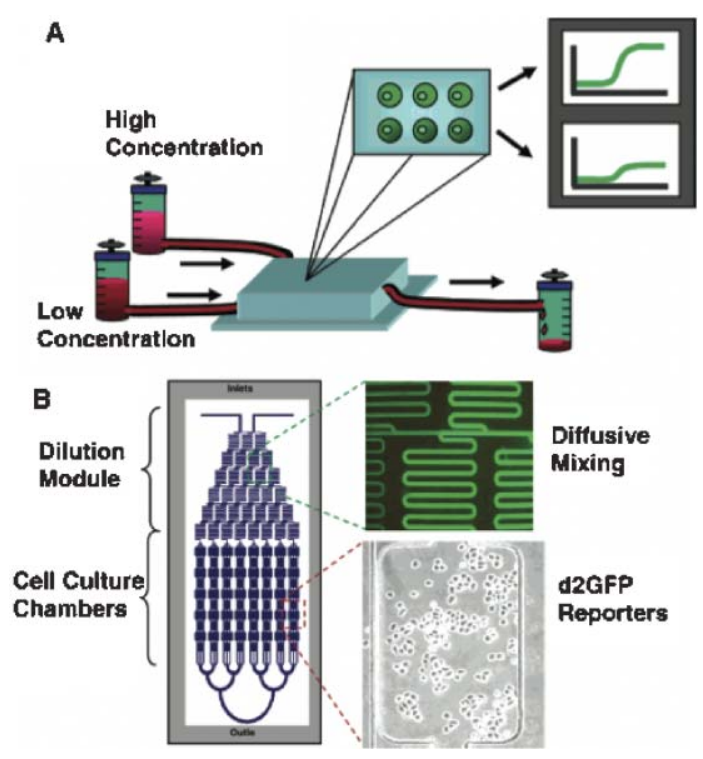

Fig. 10 Experimental setup of the Living Cell Array. Each chamber contained cells with GFP reporter proteins. The gradient generator split two inputs into eight different concentrations, which flowed into eight columns of chambers. As shown in the top right, the fluorescence intensity in each chamber would be a dynamically measurable level of specific gene expression. ${ }^{40}$ generate insufficient concentration of analyte for standard assay systems. Consequently, many biomolecular analysis procedures, discussed below, have been adapted to a microfluidic platform.

\subsection{Detection systems and devices for molecular systems biology}

Microfluidic analysis devices have been developed that can perform filtration, electrophoresis, PCR, and other methods of biomolecular analytics. In order to characterize a system, it is crucial to be able to assay target molecules after performing experimentation. Whereas fluorescent and luminescent techniques, such as GFP-tagging used in the Living Cell Array, have become widely used for investigation of molecular dynamics in systems biology, they are by no means the only tools necessary. Because of their small-scale, the microfluidic detection systems overviewed below provide the capability to autonomously process the nanoliter volumes of sample (e.g. single cell lysate) generated from aforementioned experimental platforms and within a much shorter timescale than through manual processing.

The most commonly known and appreciated method of sorting and filtering particles on a microfluidic platform is the H-Filter. ${ }^{54}$ This device takes advantage of the diffusive mixing between adjacent laminar streams in order to passively separate small particles from solutions containing both small and large particles (Fig. 11). By controlling the input stream flow rates and the channel geometry, the time allowed for diffusion of particles can be limited such that only small particles have time to diffuse to the adjacent stream, isolating them from the larger particles.

Another elegant sorting system, developed by MacDonald et al. ${ }^{55}$ sorted microscopic particles from microfluidic streams by size and refractive index with efficiency of over 95\%. They used a three-dimensional, and dynamically variable, optical lattice that caused angular deviation in flowing particles. The deflection separated two different types of particles from one laminar stream into two, at about 25 particles per second. These simple filter devices have a wide range of applications for molecular analysis, such as sorting the constituents of cell lysate and subsequently performing single molecule detection,

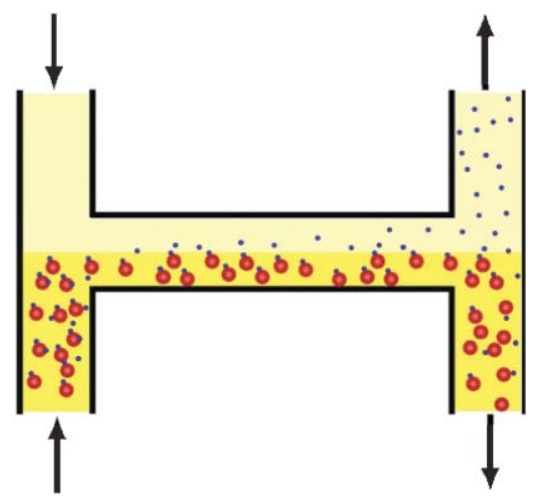

Fig. 11 Diagram of the microfluidic H-filter. Smaller particles are passively separated from larger particles and flow out in a receiver stream. $^{57}$ 
through integration with the recently developed femtoliter chamber array by Rondelez et al. ${ }^{56}$

This group was able to microfabricate a grid of chambers with volumes on the order of femtoliters. Within the chambers, they demonstrated the ability to trap single $\beta$-galactiosidase molecules and continuously visualize and quantify molecular activity with an enzyme activated fluorescence assay. Using such small quantities of molecules for study permits the necessary determination of molecular dynamic parameters on the true spatiotemporal scale at which they occur, rather than at the minimum scale of standard assays. As experiments begin generating parameters on increasingly high-resolution scales, quantitative systems biology models will begin to more accurately describe the true nature of molecular dynamics.

Similarly small numbers of molecules can be studied using microfluidic droplets. Droplets are picoliter fluid volumes usually separated by oil within a capillary or microfluidic channel. Because each droplet is completely isolated from others, each one can act as individual reactor vessels for experimentation. Through various microchannel geometries, droplet volume can be regulated, chemical concentration of droplets can be adjusted, droplets can be made to undergo fission or fusion, and a series of droplets can be sorted. ${ }^{58}$ Combining these geometrical components can be useful for creating a microfluidic device that easily manipulates thousands of reaction vessels.

An essential discovery in droplet microfluidics was the ability to perform chaotic mixing within droplets and to control the timescale of chemical reactions. ${ }^{59}$ Droplets are formed from laminar streams, and the streams remain unmixed even within a droplet. However, flowing drops through a snake-like microchannel causes controlled mixing (Fig. 12), allowing the timescale of a chemical reaction within a droplet to be controllable by the droplet distance and flow velocity. As such, the geometry of the microchannel along with controlled droplet creation can define when a reaction begins, how it evolves, and when it ends. Through fluorescence imaging of a single droplet stream, the entire timescale of a chemical reaction can be obtained with millisecond resolution. Combined with the above-mentioned droplet manipulation geometries, multiple reactions can be combined or separated at different periods in reaction time.

Using their development of controlled reactions in time, Song and Ismagilov ${ }^{60}$ demonstrated millisecond-resolution single-turnover kinetic measurements of RNase A. The device measured both fast $\left(k \sim 10^{3} \mathrm{~s}^{-1}\right)$ and slow $\left(k \sim 1 \mathrm{~s}^{-1}\right)$ kinetics and performed on-chip dilution for kinetic measurements of several concentrations without the need for any manual preparation. Since the microchannel was on a single chip and the reactions occured consistently over the entirety of its length, a single image of the channel provides the entire kinetic profile of a reaction. The microchannel could be designed to study any shape of kinetic profile by altering the density of time points. For a more detailed explanation, a thorough review on the theory behind performing droplet-based kinetic measurements is available in ref. 61.

Zheng and Ismagilov $^{62}$ developed a microfluidic droplet system for large-scale, serial screening of volumes of solution against multiple reagents. A stream of droplets was flowed

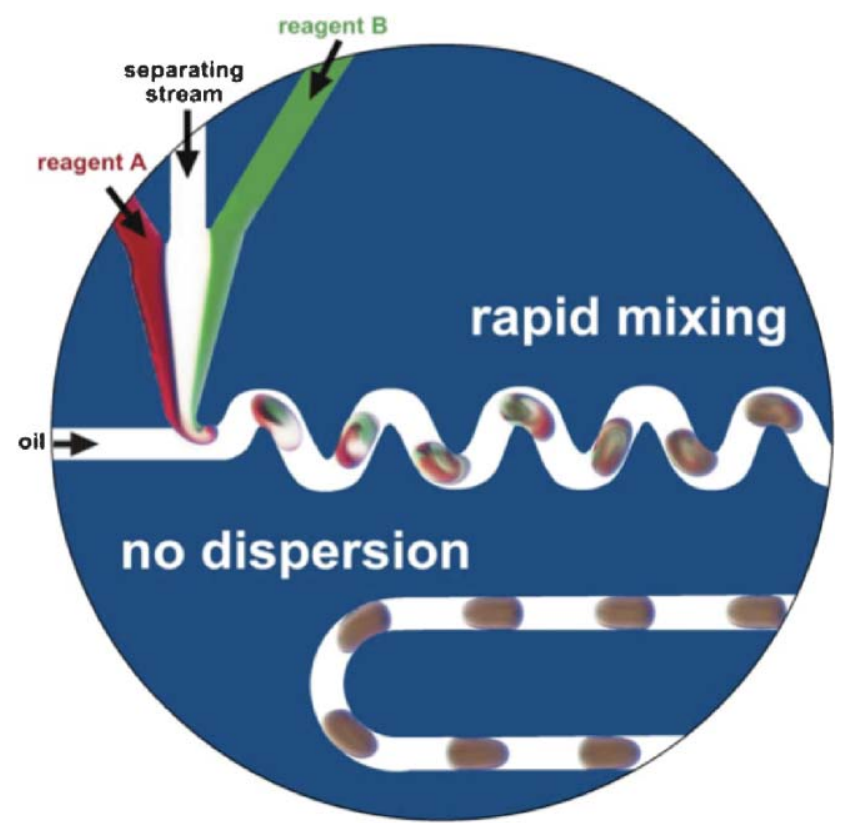

Fig. 12 Picoliter droplets formed from two reagents and separating oil. As the droplets flowed through the curved channel, chaotic mixing caused the two reagents to mix rapidly. Each droplet could act as an individual experimental reactor. ${ }^{59}$

through a microfluidic channel from a capillary and the screening solution was flowed perpendicularly into the channel to form a T-junction. At the junction, the solution flowed into the currently passing droplet, allowing observation of the reaction outcome within that particular vessel. The investigators screened a protein against a variety of crystallizing agents, with the correct agent causing protein crystallization within the droplet. The investigators also screened multiple enzymes for specific functionality. They screened alkaline phosphatase (AP), catalase, ribonuclease A (RNase A), and lysozyme against fluorescein diphosphate (FDP). FDP releases a fluorescent moiety upon hydrolysis, which is catalyzed by AP. The droplet with AP-FDP was the only one that fluoresced, as expected. An important consideration in performing such studies is protein adsorption into the microfluidic device surface, however the same group developed methods of altering droplet interfacial chemistry to actively prevent such problems. ${ }^{63}$ These developments can be further extended for the creation of artificial membranes on the droplet surface.

As with the femtoliter chambers, the discussed droplet-based microfluidic devices for kinetic parameter calculation could be used to study the constituents of cell lysates. Furthermore, their ability to precisely control and profile chemical reaction kinetics could prove to be fruitful in the high-throughput determination of reaction parameters. The ability to manipulate droplets as well as combine and separate specific reactions could provide the basis for microfluidic experimental devices that spatiotemporally emulate complex reaction networks such as those in biological systems. However, the ability to culture cells within droplets has been developed ${ }^{64}$ and could be used to test cells within droplet microreactors. The serial screening methodology discussed above could then easily be 
applied to test reagents against extremely large numbers of droplets containing individual cells at high-throughput rates.

These devices provide new and interesting means of performing molecular level characterization. More traditional tools, however, also exist on a microfluidic platform. Ewing et $a l^{65}$ developed a capillary-based electrophoresis device to dynamically study a single-cellular microenvironment. Flow from a microfluidic capillary entered a rectangular channel where the flow was subjected to electrophoresis as it translated across the channel. The investigators dynamically separated three different DNA samples (Fig. 13). They were also able to assay down to $720 \mathrm{fmol}$ of dopamine continuously over an hour. The investigators stressed the importance of single cell measurements and are developing their device in order to dynamically assay single cell secretions, however with the integration of a single cell serial feed and lysis system, it could easily be adapted to perform high-throughput single cell DNA and protein electrophoresis. Micro-scale electrophoresis is not entirely new, and very many on-chip PCR, RT-PCR, and electrophoresis devices have been developed to date; the device reviewed was chosen because of its specific demonstration of its dynamic analysis capabilities. However, Hawtin et al. ${ }^{66}$ compared on-chip PCR, RT-PCR, and electrophoresis with the conventional slab-based techniques and determined the onchip technologies to be as reliable as conventional means for high-throughput molecular systems analysis, with the added benefits of low volumes of sample and quick analysis times.

Fluorescence-activated cell sorting (FACS) is a technology by which cells can be sorted (in serial) depending on their levels of gene or protein expression. The technology has been applied to systems biology experimentation because expression levels can be quantified from a large sample of single cells. A new microfluidic FACS device has been developed that did not impose discernable stress on the cells. ${ }^{67}$ Like traditional FACS, cells flowed toward the waste stream, however if fluorescence

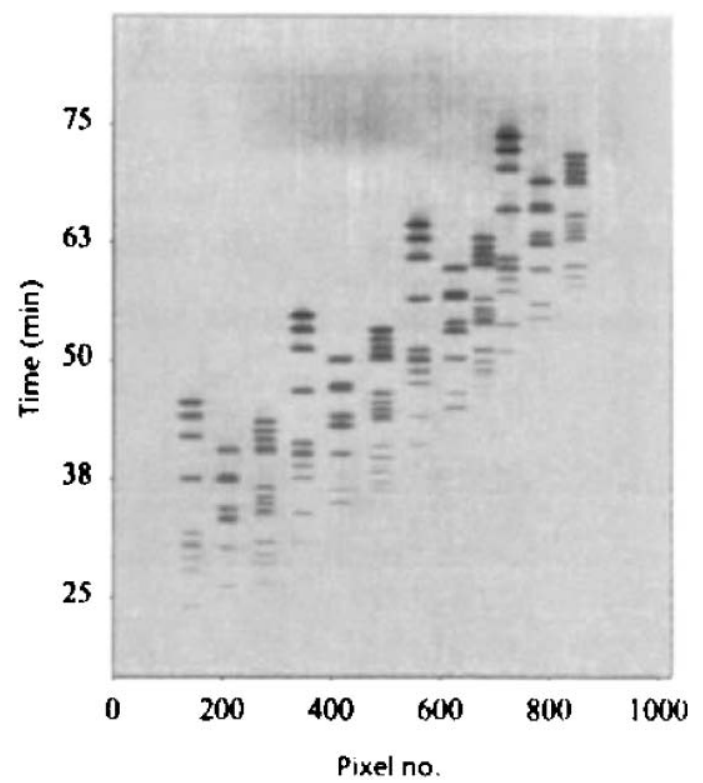

Fig. 13 DNA separations in a microfluidic rectangular channel. Separations are staggered in time from left to right, demonstrating the continuous and dynamic monitoring capabilities of the device was detected when they passed through the analysis region, a laser deflected the cells into the collection stream at 20-100 cells per s. The investigators studied the stressful effects of the device on cells by examining the expression of the heat shock and shear stress response genes, HSPA6 and FOS, respectively. Gene expression was normal, showing the method to be advantageous for sorting and studying cells without perturbing their signaling system.

Ion flux is another significant component of system models and electrophysiology experimentation plays an important role in cellular signaling. For example, the role of calcium in muscle contraction provides a direct link between signaling systems and phenotypic response. Werdich et al. ${ }^{68}$ integrated microelectrodes into a microfluidic device that was capable of isolating a single cardiac myocyte. The device trapped a single cell in a channel with recording electrodes that spread over the cell, and two drug-delivery channels directed at the cell ends (Fig. 14). The device allowed optical characterization; therefore the investigators were able to correlate visually observed contractions of the myocyte with corresponding extracellular potential spikes. The ability to locally deliver reagent was demonstrated through the perfusion of fluorescein through one channel and the observation of the subsequent intracellular fluorescence.

Classically, cellular electrophysiology is performed using the pipette-based patch-clamping technique. The process is performed serially, manually, and painstakingly slowly. Seo et al. ${ }^{69}$ designed single-cell trapping channels to develop a microfluidic patch clamping device. The device consisted of thin channels that each applied negative pressure against individual cells in order to suck in and hold onto them. The geometry used in this device facilitated many parallel experiments in a small area and allowed both optical and electrical characterization. They further performed whole-cell patch-clamp recordings on mammalian cells ${ }^{70}$ by increasing seal resistance. Measurements were performed that corresponded well with experiments using the traditional pipette-based method, with the added benefit of not needing any vibration damping equipment. They trapped mammalian $\mathrm{CHO}$ cells and recorded whole cell Kv2.1 channel currents upon incremental depolarization. They also perfused TEA into the cell reservoir, which blocks Kv2.1, recording current response to depolarization

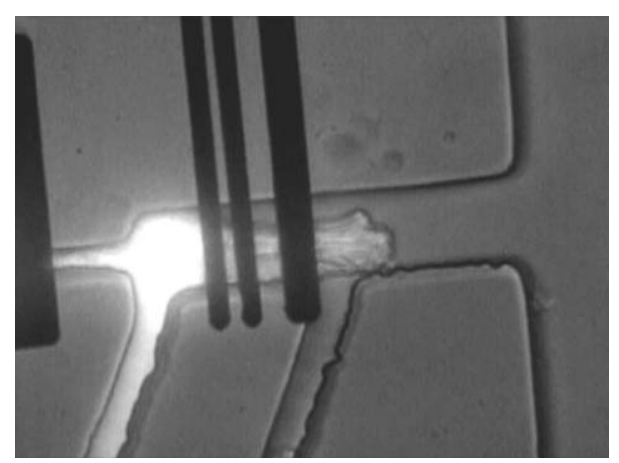

Fig. 14 Cardiac cell trapped across three recording electrodes and two drug delivery channels. The ability to perfuse drugs into the cell was demonstrated with fluorescein solution. The device was capable of both optical and electrical characterization. ${ }^{68}$ 

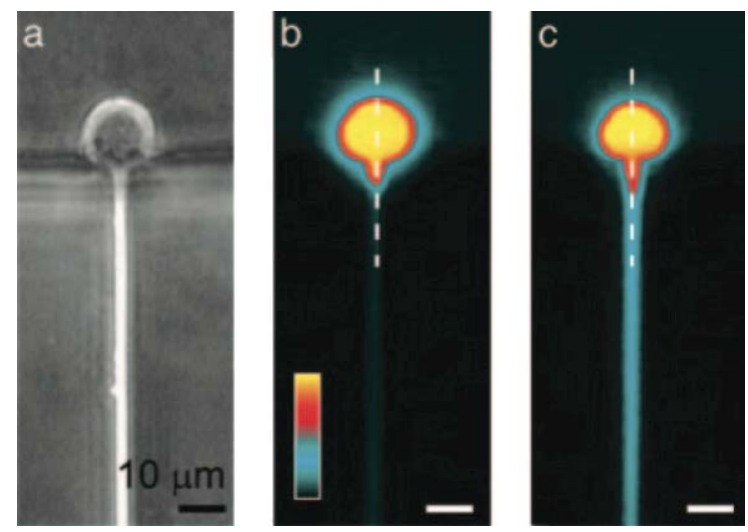

Fig. 15 (a) Single cell trapped on a microfluidic electrophysiology platform. Cells were dyed for optical characterization. The image in (b) shows the cellular deformation in the patch-clamp channel. Image (c) shows the breakage of the cellular membrane and the flow of dye into the channel after the application of a negative pressure pulse. ${ }^{70}$

before and after TEA introduction. Expectedly, there was no Kv2.1 response in the presence of TEA. The investigators measured reagent delivery time to be $\sim 20 \mathrm{~ms}$, facilitating the study of fast gating kinetics to high-speed reagent application. They also used calcium fluorescence to observe cell deformation and breakage, demonstrating the utility of the device in studying response to perturbations applied directly onto or into the cell (Fig. 15).

In addition to assaying a molecular environment for its composition, the overall molecular and chemical balance of the microenvironment may need to be controlled. Eddington et al. $^{71}$ developed a microfluidic device capable of autonomously and continuously maintaining the $\mathrm{pH}$ of the output stream in response to the input stream. The device accepts an input stream and a compensating stream, and a hydrogel undergoes volumetric change in response to the $\mathrm{pH}$ of the input, continuously adjusting the amount of the compensation stream added to the input (Fig. 16). Hydrogels responsive to temperature, light, and molecules, among others, could just as easily be used. The device is useful to isolate a specific stimulus by regulating many other factors that influence the cellular environment, especially if the cells significantly alter the environment themselves. This provides a simple and integrated control mechanism in order to regulate the cellular microenvironment, without the need for complex large-scale equipment necessary to develop a bioreactor with standard cell culture experiment.

\subsection{Integration of microfluidic components}

Even greater than the potential of the individual microfluidic devices discussed is their potential as integrated analysis systems. Experiments that are more complex could be performed with integrated microfluidic components and multiple methods of cellular study could be combined in parallel. Microfluidic components such as a flux stabilizer and a bistable flip-flop memory, analogous to their electronic circuit counterparts, have been developed ${ }^{72}$ and could be used to make increasingly controlled and integrated devices that
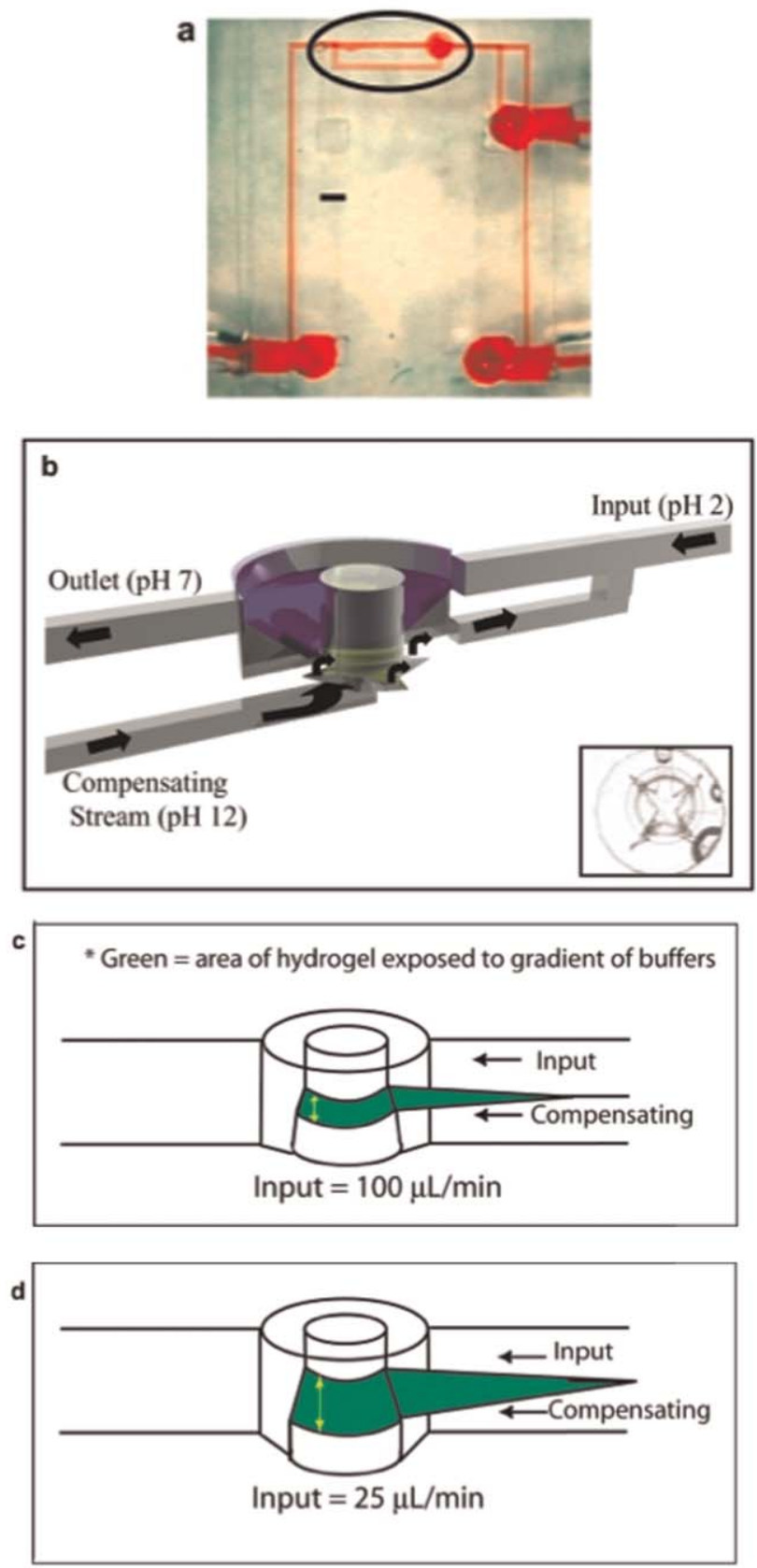

Fig. 16 (a) Picture of a self-regulation microfluidic device. Scale bar is $1 \mathrm{~mm}$. (b) Schematic and top view of the circles region in (a). (c) and (d) Schematic of the hydrogel under different input flow rates and the resulting regulated output streams. ${ }^{71}$

perform multiple operations. Microfluidic devices could also be upscaled, facilitating high-throughput experimentation.

Hong et $a l^{73}$ integrated cell isolation, lysis, DNA/mRNA purification, and recovery in a microfluidic platform. The benefit of their use of microfluidics was the ability to develop the system in parallel for simultaneous studies and the ability to analyze DNA or mRNA from down to one cell. Typical procedures for mRNA require thousands of cells, which obscure the cell-to-cell differences in gene expression. The study of single cell gene expression would be useful for identifying differentially expressed genes rather than 
commonly expressed genes. The device was composed of variously organized fluidic channels, mechanical valves, and a rotary mixing module that sequentially circulated three different flows for rapid mixing (Fig. 17).

Thorsen et $\mathrm{al}^{74}$ have remarkably demonstrated the extraordinary complexity of which microfluidics is capable. They developed microfluidic devices that performed analogous functions to electronic chips such as a comparator array and a memory storage device. The memory storage device contained 3574 valves with 1000 individually addressable picoliter chambers. The comparator contained 2056 valves for controlled loading and mixing of two different reagents into 256 individually addressable chambers (Fig. 18). Using the comparator assay, the investigators performed high-scale testing of bacteria for the expression of a specific enzyme. They loaded the device with Amplex Red, and subsequently inputted bacteria expressing an enzyme that amplified the fluorecent signal. They observed which chambers contained the bacteria by looking for the amplified signal. They further tested the device by loading both bacteria expressing the enzyme and bacteria expressing enhanced GFP instead. They successfully found that the output signal had no reliance on cells other than those expressing the amplifying enzyme. Lastly, they demonstrated the ability to selectively purge individual chambers of bacteria for collection and study.

\section{Developing an integrated microfluidic platform for systems biology}

In the past 5 years, the volume of research involving microfluidic applications in cell biology has increased dramatically. As new fabrication techniques and design principles are discovered, the repertoire of tools available to the bioengineer

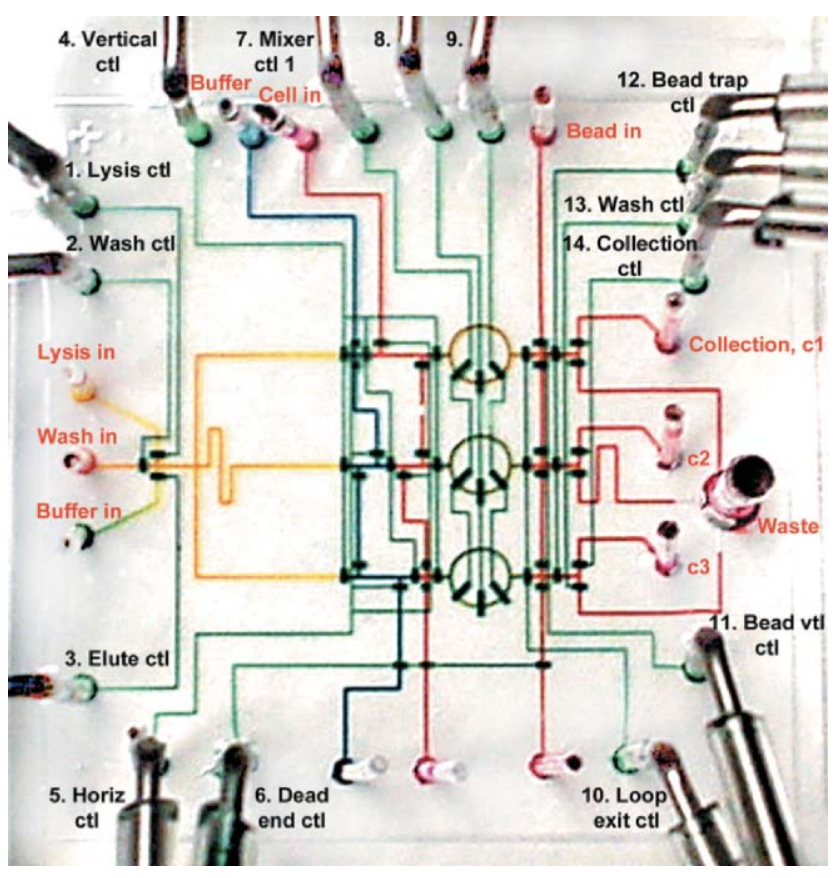

Fig. 17 Integrated DNA/mRNA purification chip with three parallel processors. The circular channels in the center are the rotary mixers and the thicker black marks are mechanical valves. ${ }^{73}$ is growing at a remarkable rate. As demonstrated in the review above, the initial steps in the field of microfluidic biology are promising. However, in order to approach the unique problems of systems biology, a clear framework needs to be adopted within the engineering community.

Two fundamental goals need to be emphasized as microengineers begin to design tools for the study of systems biology: device reliability and experimental throughput. Currently, most publications regarding microfluidic methods to investigate biology have a strong emphasis on experimental novelty. Towards this end, the majority of research is focused on "proof-of-concept" demonstrations of microfluidic devices. While this is sufficient to pique the public interest in the power of microfabricated tools for biology, the realization of practical applications for biofluidics is largely unmet and under appreciated. In order for microengineered platforms to have broader impact, it is necessary to develop methods that can be utilized by the wider systems biology community. Of primary importance is the ability to reproduce effective experimental performance. For a successful "proof-ofconcept," only a handful of data points (oftentimes collected at an overall success rate of less than $10 \%$ ) is necessary. If the same tool is designed to be used by the biological community as an experimental apparatus, the reliability of performance needs to be near $100 \%$. Unfortunately, the resources allocated for this task are fairly insignificant. Topics of research that deserve increased support include (1) optimization of design and fabrication parameters to improve device yield, (2) rigorous demonstration of experimental reproducibility, and (3) development of standardized "macro-to-micro" interfacing. These issues are of paramount importance in the field of systems biology, where it is necessary to be able to perform high throughput experimentation. Largely due to the poor reliability of current microfluidic devices, the capability of performing large-scale microfluidic experimentation is rare.

Perhaps the most relevant application of microfluidic engineering to systems biology lies in developing a high throughput live cell analysis platform. While high throughput analysis is well established on the biochemical level, there is no adequate means to control live cell perturbation on a largescale. Biofluidics integrated circuit technology offers a promising solution for this need as it has been proven for cell culture, cell-cell communication, microenvironment control, high content analysis, and large-scale integration. The proposed platform would thus be one where a large array of cells can be systematically and precisely cultured in hundreds of controlled microenvironment conditions with simultaneous quantitative measurement of cell behavior. This tool would prove to be of great value for experimentation in systems biology, and is a problem that is best addressed with microfluidic engineering.

\section{Concluding remarks}

Although most of the devices reviewed have not been used for systems biology experimentation, this paper is meant to be an overview of relevant microfluidic devices that have been developed, their use in experimentation, and their potential benefit to the field of systems biology. Microfluidics is a 


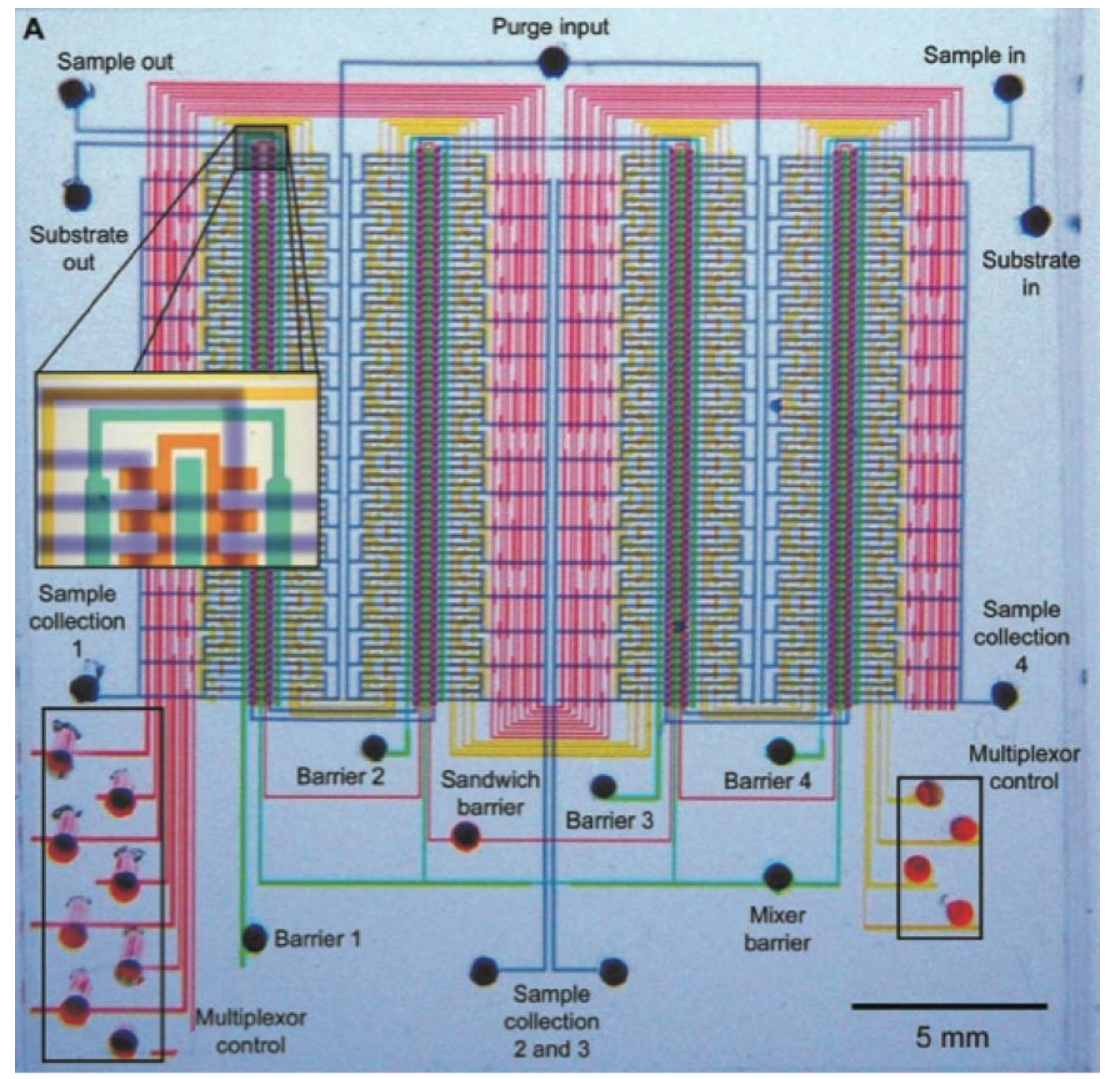

Fig. 18 Microfluidic-based comparator. The device contained 2056 valves and 256 individually addressable chambers. ${ }^{74}$

beneficial experimental basis for systems biology because: (a) Micro-scale fluid flow enables precise and high-resolution microenvironment control; (b) Microfluidic devices are generally compatible with imaging and microscopy techniques; (c) The ability to upscale and array microfluidic devices facilitates high-throughput experimentation, a necessary ability when faced with the extraordinary complexity of biological systems.

The microfluidic devices discussed in this review have already demonstrated the ability to easily address many experimental issues facing systems biology. Single cell studies have been performed in order to isolate individual cellular responses. Studies of intracellular systems can be performed on both a cell pair basis or on an entire population, with singlecell resolution. There is even strong potential for the study of cell-cell communication. Crucially, microfluidic devices enable expanded spatiotemporal characterization of molecular and cellular events. In addition, all such experimentation can be massively arrayed for high-throughput studies.

The Institute for Systems Biology (http://www.systemsbiology.org) already maintains a microfluidic facility and as the capabilities of microfluidic devices expand, their use in systems biology will quickly become as pervasive as common technologies such as DNA microarrays, while considerably expanding the experimental capabilities of the field. The density of mechanical valves within a nano-fluidic platform has been shown to follow Moore's Law, ${ }^{75}$ indicating the incredible potential of integrated microfluidic circuits for biological experimentation and automation in the near future. Additionally, the integration of microfluidic devices and biophotonic probes would add molecular-resolution sensors for further system measurement, leading toward the future hope of predictive and preventative medicine. ${ }^{76}$

\section{Acknowledgements}

We thank Dr. Kenneth Breslauer for extensive discussion and Dr. Ron Hart for critical commentary on the introduction.

\section{References}

1 H. Kitano, Systems biology: a brief overview, Science, 2002, 295, $1662-1664$.

2 T. Ideker, T. Galitski and L. Hood, A new approach to decoding life: systems biology, Annu. Rev. Genomics Hum. Genet., 2001, 2, 343-372.

3 K. Oda, Y. Matsuoka, A. Funahashi and H. Kitano, A comprehensive pathway map of epidermal growth factor receptor signaling, Mol. Syst. Biol., 2005, 1, msb4100014-E1.

4 S. Fields and R. Sternglanz, The two-hybrid system: an assay for protein-protein interactions, Trends Genet., 1994, 10, 286-292.

5 U. Korf and S. Wiemann, Protein microarrays as a discovery tool for studying protein-protein interactions, Expert Rev. Proteomics, 2005, 2, 13-26.

6 E. M. Phizicky and S. Fields, Protein-protein interactions: methods for detection and analysis, Microbiol. Rev., 1995, 59, 94-123.

7 J. P. Wikswo, 2004, Instrumentation Challenges for Systems Biology, Presented at IEEE Sensors, Vienna, Austria.

8 D. J. Beebe, G. A. Mensing and G. M. Walker, Physics and applications of microfluidics in biology, Annu. Rev. Biomed. Eng., 2002, 4, 261-286.

9 J. Knight, Honey, I shrunk the lab, Nature, 2002, 418, 474-475.

10 A. Y. Fu, C. Spence, A. Scherer, F. H. Arnold and S. R. Quake, A microfabricated fluorescence-activated cell sorter, Nat. Biotechnol., 1999, 17, 1109-1111. 
11 M. U. Kopp, A. J. Mello and A. Manz, Chemical amplification: continuous-flow PCR on a chip, Science, 1998, 280, 1046-1048.

12 T. Meyer and M. N. Teruel, Fluorescence imaging of signaling networks, Trends Cell Biol., 2003, 13, 101-106.

13 S. Takayama, E. Ostuni, P. LeDuc, K. Naruse, D. E. Ingber and G. M. Whitesides, Subcellular positioning of small molecules, Nature, 2001, 411, 1016.

14 K. Takahashi, S. N. Arjunan and M. Tomita, Space in systems biology of signaling pathways-towards intracellular molecular crowding in silico, FEBS Lett., 2005, 579, 1783-1788.

15 C. Lemerle, B. Di Ventura and L. Serrano, Space as the final frontier in stochastic simulations of biological systems, FEBS Lett., 2005, 579, 1789-1794.

16 B. M. Slepchenko, J. C. Schaff, J. H. Carson and L. M. Loew, Computational cell biology: spatiotemporal simulation of cellular events, Annu. Rev. Biophys. Biomol. Struct., 2002, 31, 423-441.

17 P. Cluzel, M. Surette and S. Leibler, An ultrasensitive bacterial motor revealed by monitoring signaling proteins in single cells, Science, 2000, 287, 1652-1655.

18 J. E. Ferrell, Jr. and E. M. Machleder, The biochemical basis of an all-or-none cell fate switch in Xenopus oocytes, Science, 1998, 280 895-898.

19 P. Uetz and R. L. Finley, Jr., From protein networks to biological systems, FEBS Lett., 2005, 579, 1821-1827.

20 T. Ideker, V. Thorsson, J. A. Ranish, R. Christmas, J. Buhler, J. K. Eng, R. Bumgarner, D. R. Goodlet, R. Aebersold and L. Hood, Integrated genomic and proteomic analyses of a systematically perturbed metabolic network, Science, 2001, 292 929-934.

21 A. G. Gilman, M. I. Simon, H. R. Bourne, B. A. Harris, R. Long, E. M. Ross, J. T. Stull, R. Taussig, H. R. Bourne, A. P. Arkin, M. H. Cobb, J. G. Cyster, P. N. Devreotes, J. E. Ferrell, D. Fruman, M. Gold, A. Weiss, J. T. Stull, M. J. Berridge, L. C. Cantley, W. A. Catterall, S. R. Coughlin, E. N. Olsen, T. F. Smith, J. S. Brugge, D. Botstein, J. E. Dixon, T. Hunter, R. J. Lefkowitz, A. J. Pawson, P. W. Sternberg, H. Varmus, S. Subramanian, R. S. Sinkovits, J. Li, D. Mock, Y. Ning, B. Saunders, P. C. Sternweis, D. Hilgemann, R. H. Scheurermann, D. DeCamp, R. Hsueh, K. M. Lin, Y. Ni, W. E. Seaman, P. C. Simpson, T. D. O'Connell, T. Roach, M. I. Simon, S. Choi, P. Eversole-Cire, I. Fraser, M. C. Mumby, Y. Zhao, D. Brekken, H. Shu, T. Meyer, G. Chandy, W. D. Heo, J. Liou, N. O'Rourke, M. Verghese, S. M. Mumby, H. Han, H. A. Brown, J. S. Forrester, P. Ivanova, S. B. Milne, P. J. Casey, T. K. Harden, A. P. Arkin, J. Doyle, M. L. Gray, T. Meyer, S. Michnick, M. A. Schmidt, M. Toner, R. Y. Tsien, M. Natarajan, R. Ranganathan and G. R. Sambrano, Overview of the Alliance for Cellular Signaling, Nature, 2002, 420, 703-706.

22 D. E. Zak, G. E. Gonye, J. S. Schwaber and F. J. Doyle, Importance of input perturbations and stochastic gene expression in the reverse engineering of genetic regulatory networks: insights from an identifiability analysis of an in silico network, Genome Res., 2003, 13, 2396-2405.

23 M. Tewari, P. J. Hu, J. S. Ahn, N. Ayivi-Guedehoussou, P. O. Vidalain, S. Li, S. Milstein, C. M. Armstrong, M. Boxem, M. D. Butler, S. Busiguina, J. F. Rual, N. Ibarrola, S. T. Chaklos, N. Bertin, P. Vaglio, M. L. Edgley, K. V. King, P. S. Albert, J. Vandenhaute, A. Pandey, D. L. Riddle, G. Ruvkun and M. Vidal, Systematic interactome mapping and genetic perturbation analysis of a C. elegans TGF-beta signaling network, Mol. Cell, 2004, 13, 469-482.

24 S. Takayama, E. Ostuni, P. LeDuc, K. Naruse, D. E. Ingber and G. M. Whitesides, Selective chemical treatment of cellular microdomains using multiple laminar streams, Chem. Biol., 2003, 10, $123-130$

25 A. Sawano, S. Takayama, M. Matsuda and A. Miyawaki, Lateral propagation of EGF signaling after local stimulation is dependent on receptor density, Dev. Cell, 2002, 3, 245-257.

26 H. S. Wiley, S. Y. Shvartsman and D. A. Lauffenburger, Computational modeling of the EGF-receptor system: a paradigm for systems biology, Trends Cell Biol., 2003, 13, 43-50.

27 E. M. Lucchetta, J. H. Lee, L. A. Fu, N. H. Patel and R. F. Ismagilov, Dynamics of Drosophila embryonic patterning network perturbed in space and time using microfluidics, Nature, 2005, 434, 1134-1138.
28 G. von Dassow, E. Meir, E. M. Munro and G. M. Odell, The segment polarity network is a robust developmental module, Nature, 2000, 406, 188-192.

29 A. Eldar, R. Dorfman, D. Weiss, H. Ashe, B. Z. Shilo and N. Barkai, Robustness of the BMP morphogen gradient in Drosophila embryonic patterning, Nature, 2002, 419, 304-308.

30 L. Giot, J. S. Bader, C. Brouwer, A. Chaudhuri, B. Kuang, Y. Li, Y. L. Hao, C. E. Ooi, B. Godwin, E. Vitols, G. Vijayadamodar, P. Pochart, H. Machineni, M. Welsh, Y. Kong, B. Zerhusen, R. Malcolm, Z. Varrone, A. Collis, M. Minto, S. Burgess, L. McDaniel, E. Stimpson, F. Spriggs, J. Williams, K. Neurath, N. Ioime, M. Agee, E. Voss, K. Furtak, R. Renzulli, N. Aanensen, S. Carrolla, E. Bickelhaupt, Y. Lazovatsky, A. DaSilva, J. Zhong, C. A. Stanyon, R. L. Finley, Jr., K. P. White, M. Braverman, T. Jarvie, S. Gold, M. Leach, J. Knight, R. A. Shimkets, M. P. McKenna, J. Chant and J. M. Rothberg, A protein interaction map of Drosophila melanogaster, Science, 2003, 302 1727-1736.

31 P. Smolen, P. E. Hardin, B. S. Lo, D. A. Baxter and J. H. Byrne, Simulation of Drosophila circadian oscillations, mutations, and light responses by a model with VRI, PDP-1, and CLK, Biophys. $J ., 2004,86,2786-2802$.

32 S. Y. Shvartsman, C. B. Muratov and D. A. Lauffenburger, Modeling and computational analysis of EGF receptor-mediated cell communication in Drosophila oogenesis, Development, 2002, 129, 2577-2589.

33 N. L. Jeon, S. K. W. Dertinger, D. T. Chiu, I. S. Choi, A. D. Stroock and G. M. Whitesides, Generation of Solution and Surface Gradients Using Microfluidic Systems, Langmuir, $2000,16,8311-8316$.

34 L. V. Dekker and A. W. Segal, Perspectives: signal transduction. Signals to move cells, Science, 2000, 287, 982-983, 985.

35 M. Affolter and C. J. Weijer, Signaling to cytoskeletal dynamics during chemotaxis, Dev. Cell, 2005, 9, 19-34.

36 C. Y. Chung, S. Funamoto and R. A. Firtel, Signaling pathways controlling cell polarity and chemotaxis, Trends Biochem. Sci., 2001, 26, 557-566.

37 P. A. Spiro, J. S. Parkinson and H. G. Othmer, A model of excitation and adaptation in bacterial chemotaxis, Proc. Natl. Acad. Sci. U. S. A., 1997, 94, 7263-7268.

38 U. Alon, M. G. Surette, N. Barkai and S. Leibler, Robustness in bacterial chemotaxis, Nature, 1999, 397, 168-171.

39 T. M. Yi, Y. Huang, M. I. Simon and J. Doyle, Robust perfect adaptation in bacterial chemotaxis through integral feedback control, Proc. Natl. Acad. Sci. U. S. A., 2000, 97, 4649-4653.

40 D. M. Thompson, K. R. King, K. J. Wieder, M. Toner, M. L. Yarmush and A. Jayaraman, Dynamic gene expression profiling using a microfabricated living cell array, Anal. Chem., 2004, 76, 4098-4103.

41 P. J. Lee, P. J. Hung, V. M. Rao and L. P. Lee, Nanoliter scale microbioreactor array for quantitative cell biology, Biotechnol. Bioeng., 2005.

42 F. Lin, W. Saadi, S. W. Rhee, S. J. Wang, S. Mittal and N. L. Jeon, Generation of dynamic temporal and spatial concentration gradients using microfluidic devices, Lab Chip, 2004, 4, 164-167.

43 S. K. W. Dertinger, D. T. Chiu, N. L. Jeon and G. M. Whitesides, Generation of Gradients Having Complex Shapes Using Microfluidic Networks, Anal. Chem., 2001, 73, 1240-1246.

44 N. Li Jeon, H. Baskaran, S. K. Dertinger, G. M. Whitesides, L. Van de Water and M. Toner, Neutrophil chemotaxis in linear and complex gradients of interleukin- 8 formed in a microfabricated device, Nat. Biotechnol., 2002, 20, 826-830.

45 F. Lin, C. M. Nguyen, S. J. Wang, W. Saadi, S. P. Gross and N. L. Jeon, Effective neutrophil chemotaxis is strongly influenced by mean IL-8 concentration, Biochem. Biophys. Res. Commun., 2004, 319, 576-581.

46 S. J. Wang, W. Saadi, F. Lin, C. Minh-Canh Nguyen and N. Li Jeon, Differential effects of EGF gradient profiles on MDAMB-231 breast cancer cell chemotaxis, Exp. Cell Res., 2004, 300, 180-189.

47 C. Neils, Z. Tyree, B. Finlayson and A. Folch, Combinatorial mixing of microfluidic streams, Lab Chip, 2004, 4, 342-350.

48 A. R. Wheeler, W. R. Throndset, R. J. Whelan, A. M. Leach, R. N. Zare, Y. H. Liao, K. Farrell, I. D. Manger and A. Daridon, 
Microfluidic device for single-cell analysis, Anal. Chem., 2003, 75, 3581-3586.

49 P. J. Lee, P. J. Hung, R. Shaw, L. Jan and L. P. Lee, Microfluidic application-specific integrated device for monitoring direct cell-cell communication via gap junctions between individual cell pairs, Appl. Phys. Lett., 2005, 86, 3902.

50 V. I. Chin, P. Taupin, S. Sanga, J. Scheel, F. H. Gage and S. N. Bhatia, Microfabricated platform for studying stem cell fates, Biotechnol. Bioeng., 2004, 88, 399-415.

51 R. Davidsson, A. Boketoft, J. Bristulf, K. Kotarsky, B. Olde, C. Owman, M. Bengtsson, T. Laurell and J. Emneus, Developments toward a microfluidic system for long-term monitoring of dynamic cellular events in immobilized human cells, Anal. Chem., 2004, 76, 4715-4720.

52 F. K. Balagadde, L. You, C. L. Hansen, F. H. Arnold and S. R. Quake, Long-term monitoring of bacteria undergoing programmed population control in a microchemostat, Science, 2005, 309, 137-140.

53 G. M. Church, 2005, From systems biology to synthetic biology, Mol. Syst. Biol., pp. msb4100007-E1.

54 J. Brody and P. Yager, Diffusion-based Extraction in a Microfabricated Structure, Sens. Actuators A: Physical, 1997, 58, 13-18.

55 M. P. MacDonald, G. C. Spalding and K. Dholakia, Microfluidic sorting in an optical lattice, Nature, 2003, 426, 421-424.

56 Y. Rondelez, G. Tresset, K. V. Tabata, H. Arata, H. Fujita, S. Takeuchi and H. Noji, Microfabricated arrays of femtoliter chambers allow single molecule enzymology, Nat. Biotechnol., 2005, 23, 361-365.

57 B. H. Weigl and K. Hedine, Lab-on-a-chip-based separation and detection technology for clinical diagnostics, Am. Clin. Lab., 2002, 21, 8-13.

58 Y. C. Tan, J. S. Fisher, A. I. Lee, V. Cristini and A. P. Lee, Design of microfluidic channel geometries for the control of droplet volume, chemical concentration, and sorting, Lab Chip, 2004, 4, 292-298.

59 H. Song, J. D. Tice and R. F. Ismagilov, A microfluidic system for controlling reaction networks in time, Angew. Chem., Int. Ed. Engl., 2003, 42, 768-772.

$60 \mathrm{H}$. Song and R. F. Ismagilov, Millisecond kinetics on a microfluidic chip using nanoliters of reagents, J. Am. Chem. Soc., 2003, 125, 14613-14619.

61 M. R. Bringer, C. J. Gerdts, H. Song, J. D. Tice and R. F. Ismagilov, Microfluidic systems for chemical kinetics that rely on chaotic mixing in droplets, Philos. Trans. A Math. Phys. Eng. Sci., 2004, 362, 1087-1104.

62 B. Zheng and R. F. Ismagilov, A microfluidic approach for screening submicroliter volumes against multiple reagents by using preformed arrays of nanoliter plugs in a three-phase liquid/liquid/ gas flow, Angew. Chem., Int. Ed. Engl., 2005, 44, 2520-2523.
63 L. S. Roach, H. Song and R. F. Ismagilov, Controlling nonspecific protein adsorption in a plug-based microfluidic system by controlling interfacial chemistry using fluorous-phase surfactants, Anal. Chem., 2005, 77, 785-796.

64 B. Schaack, B. Fouque, S. Porte, S. Combe A. Hennico, O. FilholCochet, J. Reboud, M. Balakirev and F. Chatelain, 2003, Cell Culture in Microdrops, A New Format for Cell on Chip Technology, Presented at the 7th International Conference on Miniaturized Chemical and Biochemical Analysis Systems, Squaw Valley, California, USA.

65 A. G. Ewing, P. F. Gavin, P. B. Hietpas and K. M. Bullard, Continuous separations in microfabricated channels for monitoring ultrasmall biological environments, Nat. Med., 1997, 3, 97-99.

66 P. Hawtin, I. Hardern, R. Wittig, J. Mollenhauer, A. Poustka, R. Salowsky, T. Wulff, C. Rizzo and B. Wilson, Utility of lab-on-achip technology for high-throughput nucleic acid and protein analysis, Electrophoresis, 2005, 26, 3674-3681.

67 M. M. Wang, E. Tu, D. E. Raymond, J. M. Yang, H. Zhang, N. Hagen, B. Dees, E. M. Mercer, A. H. Forster, I. Kariv, P. J. Marchand and W. F. Butler, Microfluidic sorting of mammalian cells by optical force switching, Nat. Biotechnol., 2005, 23, 83-87.

68 A. A. Werdich, E. A. Lima, B. Ivanov, I. Ges, M. E. Anderson, J. P. Wikswo and F. J. Baudenbacher, A microfluidic device to confine a single cardiac myocyte in a sub-nanoliter volume on planar microelectrodes for extracellular potential recordings, $\mathrm{Lab}$ Chip, 2004, 4, 357-362.

69 J. Seo, C. Ionescu-Zanetti, J. Diamond, R. Lal and L. P. Lee, Integrated multiple patch-clamp array chip via lateral cell trapping junctions, Appl. Phys. Lett., 2004, 84, 1973-1975.

70 C. Ionescu-Zanetti, R. M. Shaw, J. Seo, Y. N. Jan, L. Y. Jan and L. P. Lee, Mammalian electrophysiology on a microfluidic platform, Proc. Natl. Acad. Sci. U. S. A., 2005, 102, 9112-9117.

71 D. T. Eddington, R. H. Liu, J. S. Moore and D. J. Beebe, An organic self-regulating microfluidic system, Lab Chip, 2001, 1, 96-99.

72 A. Groisman, M. Enzelberger and S. R. Quake, Microfluidic memory and control devices, Science, 2003, 300, 955-958.

73 J. W. Hong, V. Studer, G. Hang, W. F. Anderson and S. R. Quake, A nanoliter-scale nucleic acid processor with parallel architecture, Nat. Biotechnol., 2004, 22, 435-439.

74 T. Thorsen, S. J. Maerkl and S. R. Quake, Microfluidic large-scale integration, Science, 2002, 298, 580-584.

75 J. W. Hong and S. R. Quake, Integrated nanoliter systems, Nat. Biotechnol., 2003, 21, 1179-1183.

76 L. Hood, J. R. Heath, M. E. Phelps and B. Lin, Systems biology and new technologies enable predictive and preventative medicine, Science, 2004, 306, 640-643. 